\title{
Kawasaki disease: pathophysiology and insights from mouse models
}

Magali Noval Rivas $\mathbb{1}^{1,2} \bowtie$ and Moshe Arditi (iD ${ }^{1,2,3 凶}$

Abstract | Kawasaki disease is an acute febrile illness and systemic vasculitis of unknown aetiology that predominantly afflicts young children, causes coronary artery aneurysms and can result in long-term cardiovascular sequelae. Kawasaki disease is the leading cause of acquired heart disease among children in the USA. Coronary artery aneurysms develop in some untreated children with Kawasaki disease, leading to ischaemic heart disease and myocardial infarction. Although intravenous immunoglobulin (IVIG) treatment reduces the risk of development of coronary artery aneurysms, some children have IVIG-resistant Kawasaki disease and are at increased risk of developing coronary artery damage. In addition, the lack of specific diagnostic tests and biomarkers for Kawasaki disease make early diagnosis and treatment challenging. The use of experimental mouse models of Kawasaki disease vasculitis has considerably improved our understanding of the pathology of the disease and helped characterize the cellular and molecular immune mechanisms contributing to cardiovascular complications, in turn leading to the development of innovative therapeutic approaches. Here, we outline the pathophysiology of Kawasaki disease and summarize and discuss the progress gained from experimental mouse models and their potential therapeutic translation to human disease.

Kawasaki disease is a systemic vasculitis that affects infants and young children ${ }^{1-3}$. Kawasaki disease is now the leading cause of acquired heart disease among children in North America, Europe and Japan ${ }^{4,5}$. The cardiovascular sequelae resulting from childhood Kawasaki disease are increasingly recognized to extend into adulthood, and the disease is no longer considered self-limiting ${ }^{6-9}$. The triggering agents for Kawasaki disease remain unidentified; however, results from our laboratory ${ }^{10,11}$ and others ${ }^{12,13}$ are consistent with the interpretation that a conventional antigen is probably responsible. Coronary arteritis and predominantly coronary artery aneurysms (CAAs) occur in up to $30 \%$ of untreated children, although this rate is reduced to $5-7 \%$ in children treated with high-dose intravenous immunoglobulin (IVIG) $)^{3,14,15}$. IVIG treatment leads to CAA regression in $60-75 \%$ of patients with Kawasaki disease ${ }^{16,17}$. However, the exact mechanisms by which IVIG reduces the rate of cardiovascular complications are unknown ${ }^{18}$. Up to $15-20 \%$ of patients with Kawasaki disease do not respond to IVIG treatment, and these individuals have an increased rate of CAA development ${ }^{3,15,19-21}$.

Kawasaki disease is associated with infiltration of the coronary artery wall by a broad variety of innate and adaptive immune cells. Immunohistochemical analysis of human post-mortem tissues shows accumulation in the arterial wall of monocytes, macrophages and neutrophils ${ }^{22,23}$, and the presence of activated $\mathrm{CD} 8^{+} \mathrm{T}$ cells ${ }^{24}$ as well as IgA $\mathrm{A}^{+}$plasma cells ${ }^{25,26}$. The release of pro-inflammatory cytokines, such as TNF and IL- $1 \beta$, by infiltrating immune cells promotes vascular endothelial cell damage and the development of CAAs ${ }^{27,28}$.

However, understanding of Kawasaki disease pathophysiology is limited by the low availability of human tissues of the disease, failure to identify specific aetiological agents triggering the disease, and incomplete understanding of the molecular and cellular mechanisms leading to cardiovascular sequelae. Therefore, experimental animal models mimicking the human features of Kawasaki disease and their translational utility have been invaluable to investigation of this disease. In this Review, we discuss advances from human and mouse studies that have contributed to an improved understanding of Kawasaki disease pathophysiology and the cellular and molecular circuitries involved in disease development. We also outline how evidence obtained from experimental mouse models of Kawasaki disease vasculitis has paved the way for the development of new efficient therapeutics to treat human Kawasaki disease.

\section{Aetiological agents}

The causative agents initiating the disease have still not been identified $>50$ years after the first description of Kawasaki disease. However, the trigger is suspected to be of viral origin and to enter the body through the 


\section{Key points}

- Kawasaki disease is a childhood systemic vasculitis leading to the development of coronary artery aneurysms; it is the leading cause of acquired heart disease in children in developed countries.

- The cause of Kawasaki disease is unknown, although it is suspected to be triggered by an unidentified infectious pathogen in genetically predisposed children.

- Kawasaki disease might not be a normal immune response to an unusual environmental stimulus, but rather a genetically determined unusual and uncontrolled immune response to a common stimulus.

- Although the aetiological agent in humans is unknown, mouse models of Kawasaki disease vasculitis demonstrate similar pathological features and have substantially accelerated discoveries in the field.

- Genetic and transcriptomic analysis of blood samples from patients with Kawasaki disease and experimental evidence generated using mouse models have demonstrated the critical role of IL- $1 \beta$ in the pathogenesis of this disease and the therapeutic potential of targeting this pathway (currently under investigation in clinical trials). co-culture supernatant of peripheral blood mononuclear cells (PBMCs) from patients with Kawasaki disease but not controls ${ }^{47,48}$. However, this result could not be replicated in later studies ${ }^{49-51}$. A peptide recognized by antibodies produced during the acute phase of Kawasaki disease has been identified in 2020 (REF. ${ }^{52}$ ). Although the protein epitopes seem similar to hepaciviruses ${ }^{53}$, further studies are required to determine the specific gene sequence from which this peptide emerges.

Altogether, the absence of consistent and reproducible studies pinpointing a specific aetiological agent suggests that Kawasaki disease is caused not by one but by multiple infectious agents. Acute Kawasaki disease is associated with infiltration of $\operatorname{IgA}^{+}$plasma cells in the respiratory tract, implying that the upper airways act as a portal of entry ${ }^{25,26}$. One suggestion is that the triggering agent might be an environmental toxin or antigen transported by wind currents ${ }^{54}$; however, this possibility cannot be rigorously assessed until precise identification of the aetiological agents is achieved ${ }^{29}$.

mucosal surfaces in the lung ${ }^{29}$ (FIG. 1). This hypothesis is supported by the seasonality of Kawasaki disease outbreaks, which is similar to that of other respiratory infections. In Japan, two seasonal peaks have been observed, one in winter and another in summer, whereas in the USA, the incidence peaks are observed during spring and winter ${ }^{30}$. Development of Kawasaki disease is age specific, with children from 6 months to 5 years of age at greatest risk ${ }^{3,30,31}$, which suggests a protective maternal passive immunity against the causative agent from birth to 6 months of age and the importance of immune system maturation in children $\geq 6$ years of age ${ }^{29}$.

The clinical features of Kawasaki disease, such as high fever, skin rash and peeling, conjunctivitis and intense release of pro-inflammatory cytokines, are reminiscent of other infectious diseases such as staphylococcal and streptococcal toxic shock syndromes ${ }^{32}$. Some studies have shown that, compared with healthy control individuals, patients with Kawasaki disease have a skewed $\mathrm{V} \beta \mathrm{T}$ cell repertoire and increased frequencies of circulating $\mathrm{V} \beta 2^{+}$and $\mathrm{V} \beta 8.1^{+} \mathrm{T}$ cells, leading to the early suggestion that a superantigen toxin might have a role in triggering Kawasaki disease ${ }^{33-35}$. However, similar results were not reproduced in later studies ${ }^{36,37}$, leading to the more generalized hypothesis that the development of Kawasaki disease might be triggered by multiple conventional antigens.

Several early studies showed reduced prevalence of antibodies to the Epstein-Barr virus (EBV) capsid antigen in Japanese children with Kawasaki disease compared with age and sex-matched control patients ${ }^{38-40}$, suggesting the involvement of an abnormal immune response to EBV in disease development. However, this difference in EBV antibody seropositivity could not be reproduced in other studies ${ }^{41-43}$. A human coronavirus was detected more frequently in respiratory secretions of patients with Kawasaki disease than in control individuals ${ }^{44}$, although, again, other studies could not replicate this finding ${ }^{45,46}$, indicating that the original association might have been coincidental. The possibility that a retrovirus is the triggering agent for Kawasaki disease has also been proposed, owing to detection of retrovirus-specific reverse transcriptase activity in the

\section{SNPs influencing susceptibility}

Although Kawasaki disease has been observed around the world and in multiple ethnic groups, geographical differences exist in incidence. The highest incidence is in Asian countries such as Korea and Japan, where it has increased over the past decades and is now 10-20 times more prevalent than in North America and Europe ${ }^{30}$. This increased susceptibility in Asian children, as well as in children with Asian ancestry living in North America $^{31}$, indicates that genetic components predispose to disease susceptibility. In Japan, siblings of children with Kawasaki disease are at increased risk of developing the disease ${ }^{55}$. Single nucleotide polymorphisms (SNPs) in multiple genes have been associated with increased susceptibility to Kawasaki disease (FIG. 1); however, mechanisms linking those SNPs with Kawasaki disease progression are not yet well understood and require more investigation.

Calcium signalling pathway. Inositol 1,4,5-trisphosphate 3-kinase C (ITPKC), a kinase that phosphorylates inositol 1,4,5-triphosphate $\left(\mathrm{IP}_{3}\right)$, is involved in many signalling processes in a wide array of cells. In T cells, $\mathrm{IP}_{3}$ is released after $\mathrm{T}$ cell receptor stimulation, thus increasing levels of intracellular $\mathrm{Ca}^{2+}$ through $\mathrm{IP}_{3}$ receptors expressed on the endoplasmic reticulum and leading to nuclear translocation of nuclear factor of activated T cells (NFAT), IL-2 production and T cell activation ${ }^{56}$. By blocking the interaction of $\mathrm{IP}_{3}$ with its receptor, ITPKC negatively regulates T cell activation. A functional SNP in ITPKC has been associated with increased risk of coronary artery lesions in Taiwanese ${ }^{57}$, Japanese and American patients with Kawasaki disease ${ }^{58}$. Mechanistically, this ITPKC polymorphism might directly contribute to $\mathrm{T}$ cell hyperactivity, and more importantly, it might promote NLRP3 inflammasome activation and increase production of IL- $1 \beta$ and IL-18 (REF. $\left.{ }^{59}\right)$. ORAI1 is a membrane-bound $\mathrm{Ca}^{2+}$ channel protein encoded by ORAI1 that is involved in the $\mathrm{Ca}^{2+}$-calcineurin-NFAT signalling pathway. Although no significant association between ORAI1 polymorphisms and Kawasaki disease 


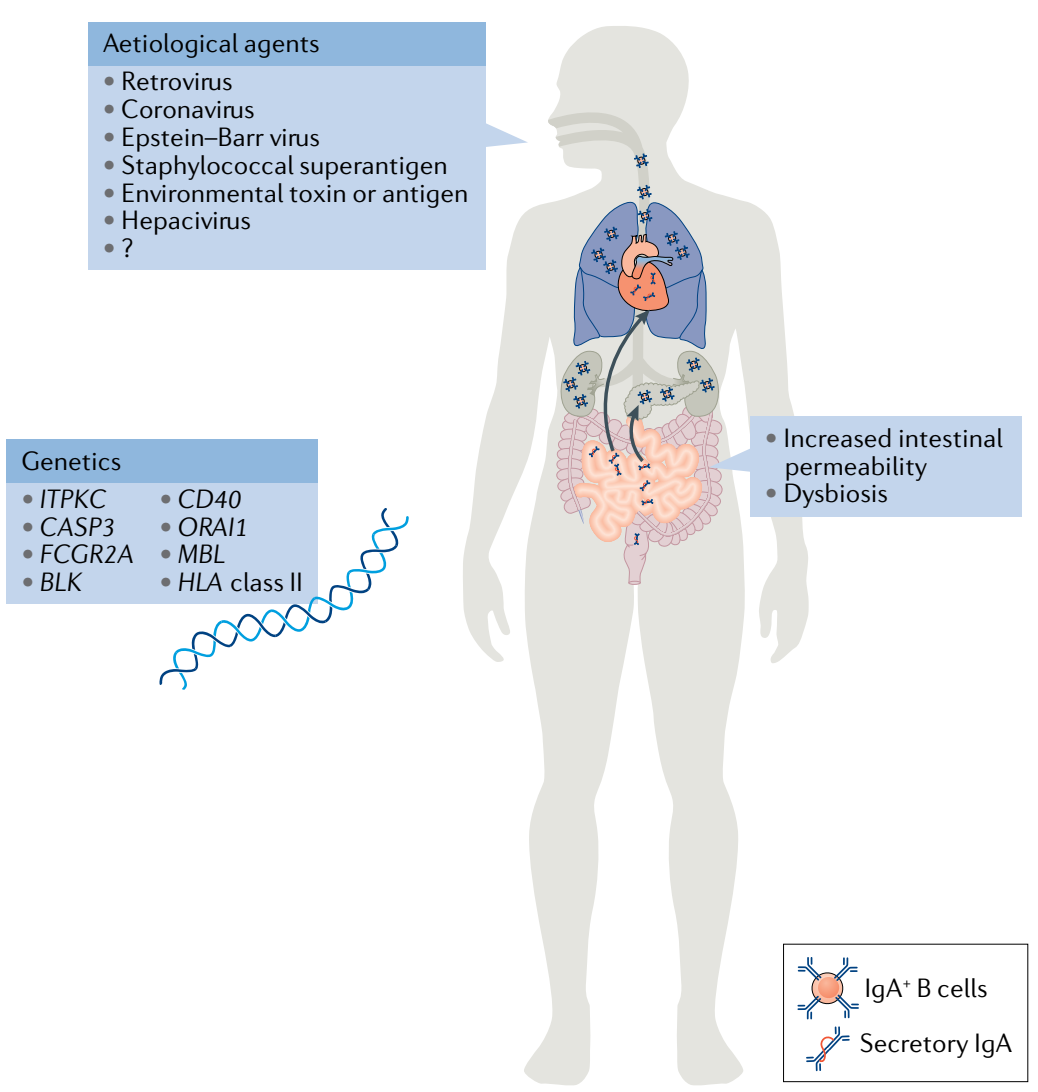

Fig. 1 | Environmental and genetic factors implicated in the development of Kawasaki disease. Different aetiological agents, from viruses to environmental toxins, have been proposed as triggering agents for Kawasaki disease; however, none has been corroborated, and the aetiological agent remains unidentified. Increased numbers of $\lg \mathrm{A}^{+}$plasma cells have been detected in the pancreas, the kidneys, the coronary artery wall and the respiratory tract of patients with Kawasaki disease. Patients with Kawasaki disease have increased concentrations of secretory $\lg A$ in their serum, indicative of defective intestinal barrier function and increased intestinal permeability. Changes in the gut microbiota composition (dysbiosis) have also been suggested to have a role in the development of Kawasaki disease. Single nucleotide polymorphisms in the genes listed have been associated with susceptibility to Kawasaki disease and disease severity. The current understanding is that Kawasaki disease is triggered in genetically predisposed children by a ubiquitous environmental stimulus that typically would not result in an uncontrolled immune response and development of vasculitis.

susceptibility or IVIG treatment response was initially reported in the Taiwanese population ${ }^{60}$, an SNP in exon 2 of ORAI1 is associated with Kawasaki disease susceptibility in the Japanese population ${ }^{61}$, and interestingly this SNP is 20 times more frequent in the general Japanese population than in the general European population $^{61}$. Another SNP in SLC8A1, which encodes the $\mathrm{Na}^{+}-\mathrm{Ca}^{2+}$ exchanger, is also associated with susceptibility to Kawasaki disease and aneurysm formation ${ }^{62}$, further highlighting the critical role of calcium signalling pathways in development of Kawasaki disease. Crucially, the $\mathrm{Ca}^{2+}$-NFAT signalling pathway is also key to intracellular $\mathrm{Ca}^{2+}$ regulation and therefore to NLRP3 inflammasome activation and IL-1 $\beta$ production ${ }^{63,64}$.

CD40 ligand. CD40 ligand (CD40L) is a protein expressed by a large array of cells including activated T cells, B cells, monocytes and platelets. CD40L receptor, CD40, is expressed by antigen-presenting cells as well as endothelial cells ${ }^{65}$. CD40 engagement is associated with cell survival, activation, proliferation and cytokine production $^{65}$. Compared with control patients with other febrile illnesses, patients with Kawasaki disease have increased $\mathrm{CD} 40 \mathrm{~L}$ expression on $\mathrm{CD} 4^{+} \mathrm{T}$ cells and platelets, which correlates with increased development of coronary artery lesions and is reduced by IVIG treatment ${ }^{66}$. An SNP in CD40L has been reported in Japanese patients with Kawasaki disease and is more frequent in male patients with coronary artery lesions than in female patients ${ }^{67}$. This polymorphism was not observed in a cohort of Taiwanese patients ${ }^{68}$; however, another SNP in the CD40 gene has been reported in an independent cohort of Taiwanese patients and is associated with increased susceptibility to Kawasaki disease and development of coronary artery lesions $s^{69}$. These results indicate a role of the CD40-CD40L pathway in the development and severity of Kawasaki disease and highlight this pathway as a potential therapeutic target.

Mannose-binding lectin. Mannose-binding lectin (MBL), a pattern recognition molecule of the innate immune system, binds the surface of pathogenic organisms and activates the complement pathway ${ }^{70}$. A polymorphism in $M B L 2$ was found to be an age-related risk factor for development of coronary artery lesions in a Dutch cohort of patients ${ }^{71,72}$. Another study in a cohort of Japanese patients with Kawasaki disease showed that codon 54 variants in MBL2 are significantly associated with susceptibility to Kawasaki disease ${ }^{73}$. Interestingly, in the Candida albicans water-soluble fraction (CAWS) mouse model of Kawasaki disease vasculitis, MBL-A and MBL-C deposition are observed in the aortic root, suggesting involvement of the MBL-dependent lectin pathway in this experimental model ${ }^{74}$. However, further studies are required to understand the pathogenic roles of those two proteins as well as their potential as therapeutic targets.

Fcy receptors. Polymorphisms in genes encoding the receptors for the Fc portion of immunoglobulins, Fcy receptors ( $\mathrm{Fc} \gamma \mathrm{Rs})$, have been associated with the development of autoimmune and infectious diseases ${ }^{75-77}$. As Kawasaki disease is considered an infectious disorder, several studies have investigated the potential association of Fc $\gamma$ R SNPs with Kawasaki disease susceptibility and the development of coronary artery lesions. In a cohort of Dutch patients, no difference in Fc $\gamma$ R SNP distribution was observed between healthy individuals and patients with Kawasaki disease, and no association was noted between SNPs in Fc $\gamma \mathrm{R}$ genes and Kawasaki disease susceptibility ${ }^{78}$. However, a later study with $>2,000$ patients with Kawasaki disease and 9,000 control patients from multiple independent cohorts across different populations highlighted a Kawasaki diseaseassociated polymorphism in the FCGR2A locus, which encodes Fc $\gamma$ RIIA (CD32a), a member of the family of IgG receptors ${ }^{79}$. This polymorphism has important implications as the standard of care for Kawasaki disease is IVIG, a pool of plasma IgG that interacts with Fc $\gamma$ Rs on immune cells. Interestingly, $15-20 \%$ of patients with Kawasaki disease have IVIG-resistant disease and 

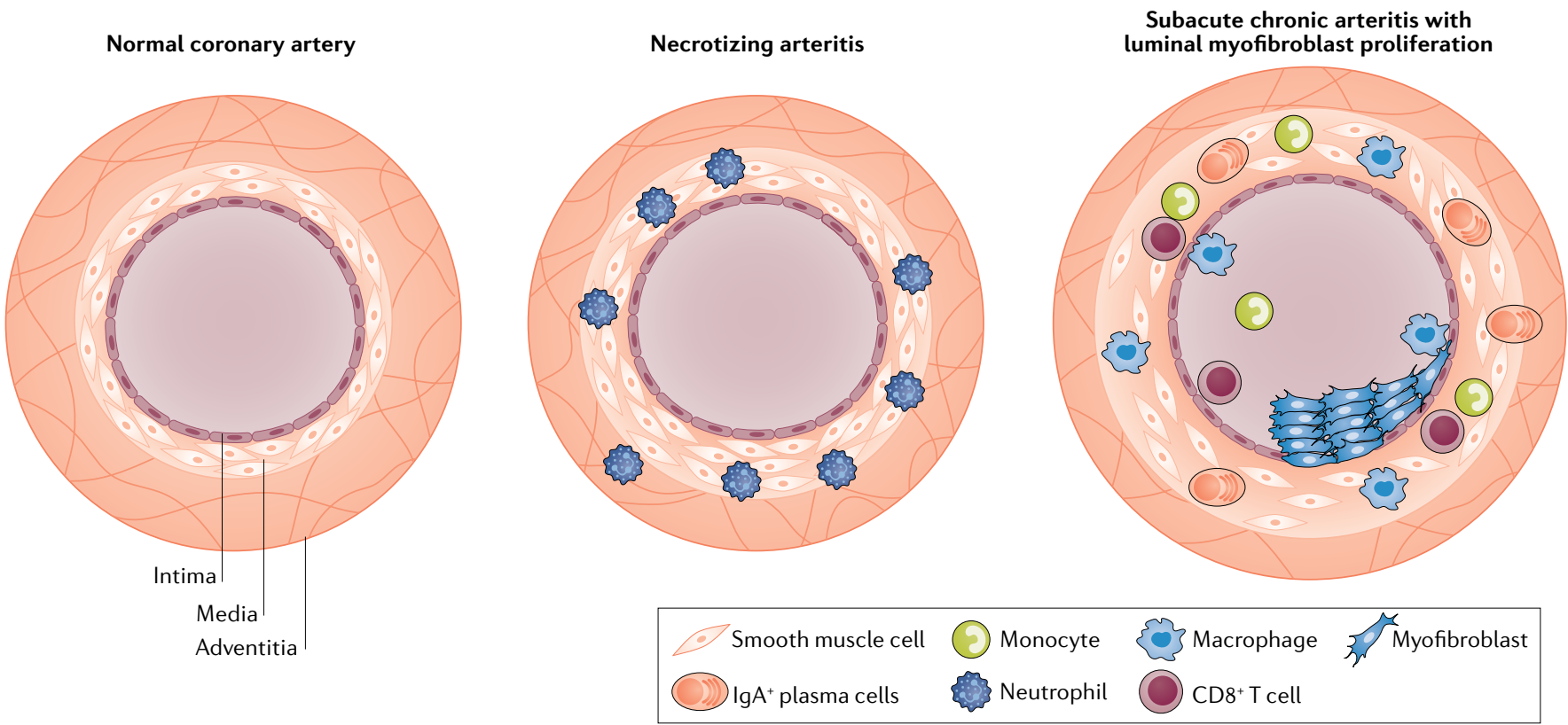

Fig. 2 | Pathophysiology of Kawasaki disease vasculitis. The normal coronary artery is composed of three general layers: the tunica intima, tunica media and tunica adventitia. The intima is mainly composed of endothelial cells, the media of smooth muscle cells and the adventitia of loose connective tissue. In Kawasaki disease, necrotizing arteritis develops in the first 2 weeks of the disease and is associated with neutrophilic infiltration, which gradually destroys the intima, media and some portions of the adventitia of the coronary artery. $\mathrm{CD} 8^{+} \mathrm{T}$ cells, $\lg \mathrm{A}^{+}$plasma cells, monocytes and macrophages compose the inflammatory infiltrate during subacute chronic arteritis. These cells release pro-inflammatory cytokines such as IL-1 $\beta$ and TNF, which contribute to luminal myofibroblast proliferation, in which myofibroblasts, mainly derived from smooth muscle cells, and their matrix products progressively obstruct the coronary lumen.

require another round of IVIG treatment or the use of adjunctive therapies ${ }^{15,19,20,80}$. The exact mechanisms by which IVIG mediates its therapeutic effect and how IVIG resistance develops remain unknown, and the potential involvement of this Fc $\gamma$ RIIA polymorphism in IVIG resistance requires further investigation.

\section{Pathophysiology of Kawasaki disease}

The innate immune response. The immune response associated with Kawasaki disease is complex and involves the activation and infiltration of the coronary artery wall by both innate and adaptive immune cells (FIG. 2). On the basis of studies of post-mortem tissue from patients with Kawasaki disease, Kawasaki disease vascular pathology has been classified into three sequential linked pathological processes ${ }^{81}$. Necrotizing arteritis develops in the first 2 weeks of the disease and is associated with neutrophilic infiltrations, which gradually destroy the coronary artery intima, media and some portions of the adventitia. Alarmins from the S100 protein family, which are present in the cytoplasm of neutrophils, monocytes and macrophages ${ }^{82}$, also participate in this inflammatory process. Concentrations of circulating S100A8/A9 heterodimers (calprotectin) and S100A12 are substantially higher in patients with Kawasaki disease during the acute phase than in control patients with other febrile illnesses and decline after IVIG treatment ${ }^{83-85}$. After the acute phase of Kawasaki disease, plasma concentrations of S100A8/A9 heterodimers only remain elevated in patients with giant $\mathrm{CAAs}^{84}$, highlighting its potential utility as a biomarker to monitor long-term persistence of inflammation. S100A12 also contributes to the acute inflammatory response by directly stimulating monocytes to produce IL- $1 \beta$, which in turn activates coronary endothelial cells ${ }^{85}$. Necrotizing arteritis might result in the formation of CAAs and is followed by two other processes, subacute or chronic vasculitis and luminal myofibroblast proliferation (LMP), which occur simultaneously and might be observed for months to years after disease onset ${ }^{81}$. The inflammatory infiltrates are composed of $\mathrm{CD}^{+} \mathrm{T}$ cells, $\mathrm{IgA}^{+}$plasma cells, eosinophils and macrophages, which release pro-inflammatory cytokines contributing to cardiovascular pathology. Meanwhile, myofibroblasts, mainly derived from smooth muscle cells, and their matrix products progressively obstruct the coronary lumen ${ }^{81}$ (FIG. 2). Persistent subacute and chronic vasculitis and LMP can lead to stenosis and thrombosis after acute illness ${ }^{6,9}$.

Matrix metalloproteinases. Matrix metalloproteinases (MMPs; zinc-dependent endopeptidases that degrade extracellular matrix components) are known to have an important role in both inflammation and tissue remodelling processes ${ }^{86}$. Increased expression and activity of a diverse set of MMPs has been demonstrated in acute Kawasaki disease ${ }^{87-89}$. The expression levels of MMP3 and MMP9, both known to mediate vascular smooth muscle cell migration and neointimal formation ${ }^{90}$, are increased in patients with Kawasaki disease ${ }^{91}$, and the circulating levels of these MMPs correlate with the development of CAAs in these patients ${ }^{22}$. MMP3 SNPs are also associated with the development of CAAs ${ }^{88}$, and 
this protease is considered to be a driving factor allowing IL-1-induced signalling to lead to migration of vascular smooth muscle cells and their transition to proliferating myofibroblasts ${ }^{93-95}$. Whereas MMP9 has been studied and implicated in elastin breakdown in the Lactobacillus casei cell wall extract (LCWE)-induced Kawasaki disease mouse model ${ }^{96,97}$, information about the role of MMP3 in this mouse model is lacking.

MicroRNAs. MicroRNAs (miRNAs; a class of small non-coding RNAs that regulate mRNA expression) are emerging as critical gene regulators in a host of cellular processes, including inflammation ${ }^{98}$. Of human coding genes, $60-70 \%$ are estimated to be regulated by miRNAs ${ }^{99}$. Several studies attempting to discover Kawasaki disease biomarkers have found that the miRNA profiles of serum exosome or coronary artery tissues are associated with acute Kawasaki disease $\mathrm{e}^{100-104}$. These miRNAs include miR-23a ${ }^{100-103}$, miR-27 ${ }^{100}$, miR-223 (REFS ${ }^{100-103}$ ) and miR-145 (REF. ${ }^{103}$ ). These miRNAs might provide clues as to the molecular mechanisms involved in the development of the cardiovascular lesions associated with Kawasaki disease. For example, miR-145 is highly expressed in vascular smooth muscle cells and has been reported to promote their switching to neointimal proliferating cells ${ }^{105,106}$ and to regulate the transforming growth factor- $\beta$ signalling pathway ${ }^{103}$. Increased levels of miR-23a contribute to cardiomyocyte apoptosis and may promote inflammatory responses by blocking macrophage autophagy activity ${ }^{107,108}$. However, improved understanding and characterization of the molecular and cellular mechanisms underlying the different roles of miRs during Kawasaki disease require further studies with animal models.

Myocarditis. Most attention in Kawasaki disease research and clinical practice has focused on the development of CAAs and long-term complications of coronary artery stenosis and ischaemia ${ }^{109}$. However, the subacute and chronic inflammation of Kawasaki disease is also associated with the development of myocarditis $^{3,6,110-112}$. Myocarditis has been described as the 'hidden face of the moon' in Kawasaki disease ${ }^{110}$. Reports indicate that myocarditis occurs frequently during acute Kawasaki disease ${ }^{111}$, and serial myocardial biopsy studies have documented that histological myocarditis develops in the majority of patients with Kawasaki disease, even in the absence of coronary aneurysms ${ }^{113,114}$. More recent data indicate that myocardial inflammation can be documented in $50-70 \%$ of patients using gallium citrate $\left({ }^{67} \mathrm{Ga}\right)$ scans and technetium-99 $\left({ }^{99 \mathrm{~m} T c}\right)$ labelled white blood cell scans ${ }^{115}$. Another study has shown that myocardial inflammatory changes and myocardial oedema in Kawasaki disease occur even before coronary artery abnormalities and without concurrent ischaemic damage $^{112}$.

Myocarditis in Kawasaki disease tends to develop early, and acute left ventricular dysfunction is generally transient and responds readily to anti-inflammatory treatment ${ }^{116}$. However, Kawasaki disease myocarditis might be associated with fatal arrhythmias in infants, and in certain cases might lead to long-term complications including myocardial fibrosis ${ }^{81,117}$. Therefore, myocarditis during Kawasaki disease and its potential consequences deserve serious investigation, and long-term studies into late adulthood are needed.

Complement and immune complexes. Kawasaki disease affects small and medium sized vessels, particularly the coronary arteries; however, dilatations and aneurysms can occur systemically, including in the axillary, subclavian, brachial, renal and iliac arteries as well as the abdominal aorta ${ }^{23,118-120}$. Post-mortem findings have revealed that $73 \%$ of patients with Kawasaki disease have renal artery involvement and acute kidney injury ${ }^{121}$ involving glomerulonephritis with intracapillary changes and deposition of immune complex composed of IgA and complement component 3 (C3 $)^{22,122,123}$. These findings are comparable to those in two other human vasculitis diseases, IgA vasculitis (IgAV) and IgA nephropathy (IgAN), which are similarly characterized by IgA immune complexes with $\mathrm{C} 3$ deposition in kidney glomeruli (see below). Increased concentrations of circulating $\operatorname{Ig} \mathrm{A}$ and secretory $\operatorname{IgA}(\operatorname{sg} \mathrm{A})$ have been reported in the serum of children with Kawasaki disease during the acute phase ${ }^{124}$. $\operatorname{IgA}^{+}$plasma cells are present in the coronary artery wall and in non-vascular tissues, such as the kidney, trachea and pancreas of patients with Kawasaki disease ${ }^{25,26}$. This IgA response is oligoclonal, seems to be antigen driven and might be caused by Kawasaki disease-triggering agents ${ }^{125,126}$.

The IL-1 signalling pathway. Evidence from mouse models of Kawasaki disease $\mathrm{e}^{11,127,128}$, as well as transcriptome analysis performed on whole blood of patients with Kawasaki disease during the acute or convalescent phase ${ }^{129,130}$, demonstrate the involvement of innate immune cells and inflammasome overactivation throughout the acute phase of the disease. In vitro cultured PBMCs isolated from patients with Kawasaki disease spontaneously release IL- $1 \beta$ into the supernatant, and this process is substantially reduced after IVIG treatment ${ }^{28}$. Serum concentrations of both IL- $1 \beta$ and IL-18 are also higher in children with acute Kawasaki disease than in control patients with other febrile illnesses, and markedly decrease during the convalescent phase ${ }^{59}$, supporting the concept of activation of the NLRP3 inflammasome complex. Similarly, IL-1 and NLRP3-related gene transcripts are upregulated in PBMCs from patients with acute Kawasaki disease and are decreased during the convalescent phase of the disease $^{59}$, and an $I L 1 B$-gene-related signature is associated with acute phase disease and IVIG resistance ${ }^{130}$. Furthermore, a study has shown that differential expression of IL- $1 \beta$ and related signalling genes might have a role in mediating the sex-based differences seen in patients with Kawasaki disease ${ }^{131}$. In the LCWE mouse model of Kawasaki disease, the activation of caspase 1, IL- $1 \alpha$ and IL- $1 \beta$ is key to the development of coronary arteritis, aneurysms, myocarditis and abdominal aorta aneurysms $s^{127,128,132}$. IL-1 has the capacity to expand and promote the differentiation of antigen-specific $\mathrm{CD}^{+}$ $\mathrm{T}$ cells ${ }^{133}$, and indeed the frequencies of circulating $\mathrm{CD} 4^{+}$ and $\mathrm{CD}^{+} \mathrm{T}$ cells are increased in patients with Kawasaki 
disease ${ }^{134}$. Infiltrations of mature dendritic cells as well as activated cytotoxic $\mathrm{CD} 8^{+} \mathrm{T}$ cells have been reported in arterial layers of coronary aneurysms ${ }^{24,135}$. Therefore, blocking the NLRP3-IL-1 $\beta$ pathway seems to be a valid therapeutic option in Kawasaki disease.

\section{Role of the gastrointestinal tract}

Intestinal permeability. The intestinal barrier has a critical role in maintaining intestinal homeostasis and health by preventing harmful organisms and luminal antigens from entering the circulation. A dysfunctional intestinal barrier, characterized by increased intestinal permeability, is recognized as a pathogenic factor in many inflammatory diseases ${ }^{136}$. In Kawasaki disease, abdominal pain, diarrhoea and vomiting are often observed at the onset of acute illness, affecting up to $60 \%$ of diagnosed patients and indicating that the gastrointestinal tract is also affected ${ }^{4,137-140}$. A multicentre study of $>300$ patients revealed that gastrointestinal manifestations at onset of disease complicate diagnosis, delay adequate treatment and correlate with IVIG resistance and severity of CAAs ${ }^{141}$. Immunohistochemical studies have revealed higher numbers of activated $\mathrm{CD}^{+} \mathrm{T}$ cells and macrophages along with lower numbers of $\mathrm{CD}^{+} \mathrm{T}$ cells in the jejunum lamina propria in patients with Kawasaki disease than in control patients with diarrhoea from cows' milk protein intolerance ${ }^{142}$. However, these cellular abnormalities are specific to the acute phase of the disease and return to normal during the convalescent phase $\mathrm{e}^{142}$. IgA $\mathrm{Ig}^{+}$plasma cells have also been observed in a variety of different vascular and non-vascular tissues in patients with Kawasaki disease $^{26}$, and patients with Kawasaki disease also have increased concentrations of $\operatorname{sig} \mathrm{A}$, which is produced at the intestinal mucosal surface, in their serum ${ }^{124}$. These studies indicate that the gastrointestinal tract is affected during Kawasaki disease and that mucosal immune activation might compensate and protect from defective intestinal barriers.

The role of gut-related immunity in the induction of inflammation in organ systems distant from the gut has been the subject of intensive investigation. We have observed increased intestinal permeability and a dysregulated intestinal immune response characterized by increased numbers of $\operatorname{IgA}^{+} \mathrm{B}$ cells in the Peyer's patches in the LCWE-induced mouse model of Kawasaki disease ${ }^{143}$ (FIG. 3). In this model, the excessive IL- $1 \beta$ release associated with LCWE injection acts on intestinal epithelial cells to open tight junctions, and administration of IVIG or pharmacological agents that block intestinal permeability significantly reduces disease development ${ }^{143}$. Altogether, these observations link increased intestinal permeability and defective intestinal barrier function with systemic IL- $1 \beta$ release in Kawasaki disease.

The intestinal microbiome. Despite the strong connection between the intestinal microbiome and development of cardiovascular diseases ${ }^{144,145}$, only a few studies have investigated the role of the intestinal microbiome during development of Kawasaki disease or treatment resistance. Microbiological culture-based methods demonstrated that, compared with healthy control individuals, patients with Kawasaki disease have a different intestinal microbiota composition characterized by a lower incidence of the Lactobacillus genus ${ }^{146,147}$ and increased Streptococcus and Staphylococcus ${ }^{148}$ species. Lactobacilli have been reported to prevent diarrhoeal disorders ${ }^{149,150}$ and to improve intestinal barrier function by increasing the expression of intestinal tight junctions ${ }^{151,152}$, enhancing the intestinal mucus layer ${ }^{153}$ and modulating the intestinal microbiota composition $^{154}$. Lactobacilli have also been shown to boost innate and immune functions against a variety of bacterial infections ${ }^{155-157}$, and their disappearance during acute Kawasaki disease might lead to the blooming of other bacterial pathogens, which might further promote intestinal barrier dysfunction and inflammation. Intriguingly, a retrospective study of 364 patients with Kawasaki disease showed that children who received microbiomealtering antibiotics in the week before Kawasaki disease diagnosis were substantially more likely to have IVIGresistant disease than those who did not receive antibiotics ${ }^{158}$. Antibiotics alter the abundance, taxonomic richness and diversity of the bacterial ${ }^{159,160}$ as well as fungal ${ }^{161}$ intestinal microbiome, and those alterations might persist from weeks to years after treatment discontinuation ${ }^{159,160,162}$. A longitudinal metagenomic study of faecal samples derived from patients with Kawasaki disease showed a marked increase of five Streptococcus spp. during the acute phase of Kawasaki disease ${ }^{163}$; however, all patients in that study were treated with antibiotics in the early stage of disease, therefore this observation might be reflective of antibiotic-induced dysbiosis and not Kawasaki disease itself. Nonetheless, how this intestinal dysbiosis occurs and how its effect on intestinal permeability affects the development of cardiovascular lesions during Kawasaki disease vasculitis remains unknown and under-appreciated.

\section{Link with $\lg \mathrm{A}$ vasculitis}

IgAV, or Henoch-Schönlein purpura, is an IgA-mediated necrotizing vasculitis resulting in fibrinoid destruction of the affected small vessels. Renal involvement, characterized by IgA deposition in the kidney glomeruli, is also observed in $\operatorname{IgAV}^{164}$. IgAV nephritis is closely related to another glomerular disease, IgAN, wherein accumulation and deposition of IgA and IgA immune complexes in the kidney glomerular mesangium drive glomerular inflammation ${ }^{165}$. As IgA is mainly found at mucosal surfaces, a 'gut-kidney axis', influenced by a mix of genetic, microbial and dietary factors, has been suggested to be involved in the development of both IgAN ${ }^{166}$ and IgAV in paediatric and adult patients ${ }^{167}$. We have demonstrated that the LCWE-induced mouse model of Kawasaki disease vasculitis is associated with the deposition of IgA and IgA-C3 immune complexes in vascular tissues, such as the inflamed coronary artery and abdominal aorta ${ }^{143}$. Deposited IgA and IgA-C3 immune complexes might result in overactivation of the immune cells present in the cardiovascular lesions and subsequent amplification of inflammation ${ }^{143}$. Substantial evidence indicates that immune complexes might promote vascular damage during human Kawasaki disease through the 

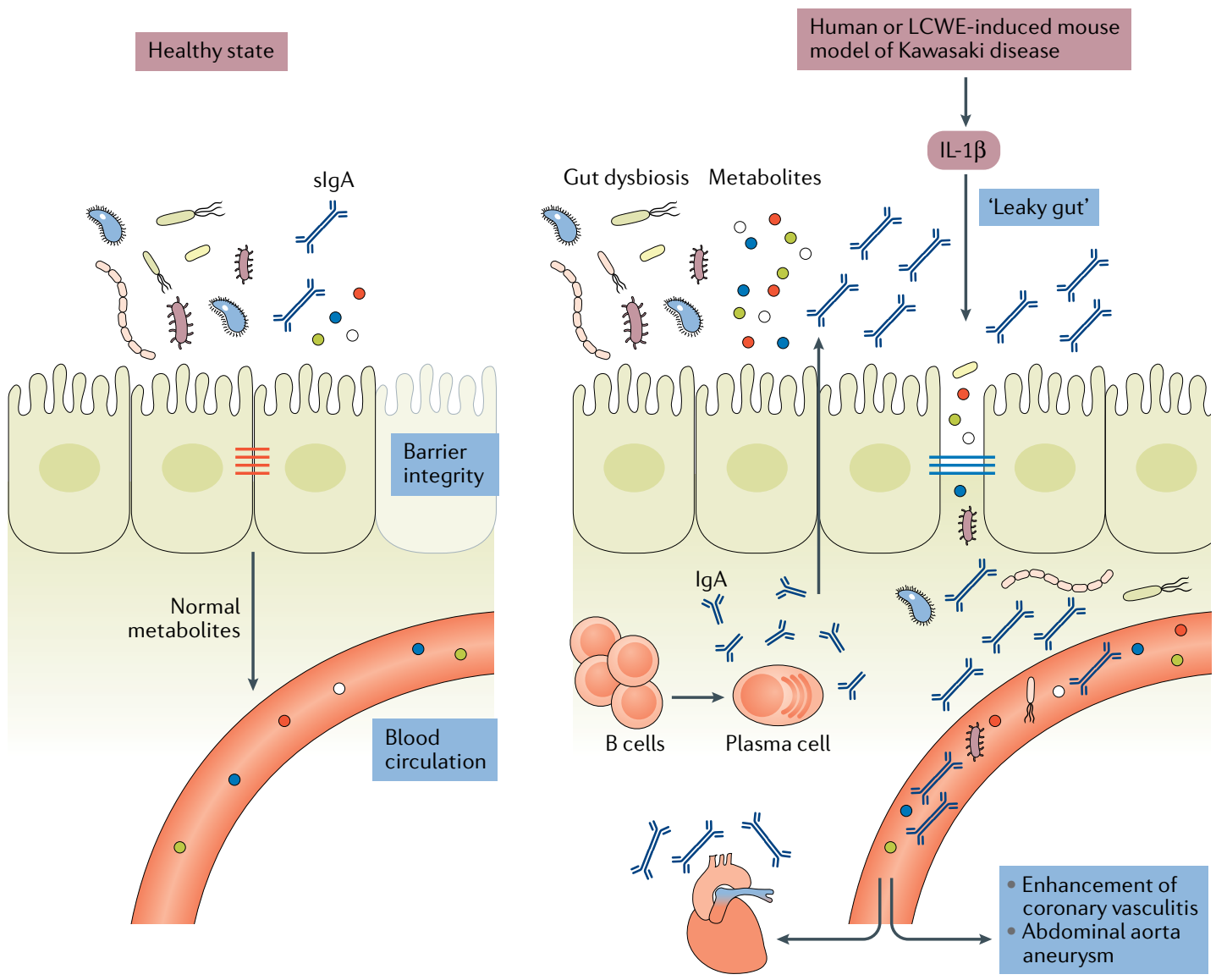

Fig. 3 | Existence of a 'gut-vascular' axis in Kawasaki disease vasculitis. In healthy individuals, intestinal epithelial cells are sealed together by intestinal tight junctions, and the intestinal epithelium acts as a barrier that prevents the passage of commensal bacteria and pathogens while permitting intercellular flux of ions, molecules and metabolites. Lactobacillus casei cell wall extract (LCWE)-induced Kawasaki disease vasculitis and human Kawasaki disease are associated with increased IL-1 $\beta$ production, which leads to decreased expression of intestinal tight junctions, resulting in increased intestinal permeability. Differences in intestinal microbiota composition have been observed in patients with Kawasaki disease, and intestinal dysbiosis might contribute further to the inflammatory process. LCWE injection is also associated with a dysregulated intestinal immune response characterized by increased numbers of $\lg \mathrm{A}^{+} \mathrm{B}$ cells in the gastrointestinal tract and elevated secretory $\lg \mathrm{A}(\mathrm{s} \lg \mathrm{A}$ ) concentrations. Intestinal barrier dysfunction results in slgA leakage to the systemic circulation and pathogenic IgA-C 3 immune complex deposition in the vascular tissues.

activation and aggregation of platelets, the release of vasoactive mediators, and the subsequent recruitment of neutrophils and leukocytes to the site of inflammation (reviewed elsewhere ${ }^{168}$ ).

Interestingly, we have also observed IgA and C3 deposition in the kidney glomeruli of LCWE-injected mice developing Kawasaki disease ${ }^{143}$, and immune complex-mediated nephropathy has also been observed in Kawasaki disease ${ }^{123}$. However, to date IgA deposition has not been reported in CAAs of patients with Kawasaki disease. Given that availability of human tissue samples is limited, and those that are available are usually collected at the end stage of the disease, they might not be representative of active Kawasaki disease pathological features, and further studies are warranted. Like Kawasaki disease, IgAV develops mostly in children, affects males more than females, is more predominant in Asian countries such as Japan and Korea, and is also associated with abdominal pain, diarrhoea, skin rash and IgA deposition in the affected small vessels ${ }^{169}$.
IgAN also shares pathological features with Kawasaki disease, such as increased intestinal permeability, low to moderate intestinal inflammation associated with activation of inflammatory cells in the small intestinal mucosa and colocalization of sIgA-complement in the glomerular mesangium ${ }^{165,170}$. Moreover, a polymorphism in the promoter of the lipopolysaccharide (LPS) receptor CD14 (CD14/159) is associated with coronary artery abnormalities in patients with Kawasaki disease ${ }^{171}$ and has been linked to progression of IgAN to more severe renal disease ${ }^{172}$. IL- $1 \beta$ has a key pathogenic role during Kawasaki disease and also seems to be implicated in renal complications related to $\operatorname{IgAV}^{173}$ and $\mathrm{IgAN}^{174}$. Altogether, given that Kawasaki disease shares clinical features and pathological mechanisms with both IgAV and IgAN, it is possible that Kawasaki disease is a form of IgAV. Similarly, treatments that have shown efficacy in Kawasaki disease, such as anakinra and IVIG, might be suitable and useful for treating $\operatorname{IgAV}^{175}$ and IgAN. 


\section{Mouse models of Kawasaki disease}

The lack of identification of specific aetiological agents and incomplete understanding of the molecular mechanisms involved in Kawasaki disease cardiovascular pathology have delayed the development of targeted and effective treatment options for this disease. In addition, the limited availability of tissue samples from patients with Kawasaki disease has considerably impeded progress in understanding the pathogenesis of the disease, making the availability of relevant animal models of Kawasaki disease extremely valuable. Kawasaki disease vasculitis can be induced in mice by injection of cell wall components from L. casei ${ }^{176}$, C. albicans ${ }^{177}$ or nucleotide-binding oligomerization domain containing 1 (Nod1) ligand ${ }^{178}$ (TABLE 1). These mouse models of Kawasaki disease have accelerated research and have enhanced understanding of the pathogenesis of this disease. However, no animal model perfectly recapitulates human disease. Particularly in the context of Kawasaki disease, given that the aetiology remains unknown, researchers must exercise caution in interpreting results based on experimental models and confirm findings in patient cohorts. Nevertheless, even though the extrapolation of preclinical mouse data to humans is far from straightforward, mouse models are still invaluable tools to study certain pathological aspects of human inflammatory diseases and gain mechanistic insights.

The LCWE mouse model. L. casei is a Gram-positive bacteria that colonizes the gastrointestinal and urogenital tracts of both human and animals ${ }^{179}$. More than 35 years ago, Lehman et al. ${ }^{180}$ demonstrated that a single intraperitoneal injection of LCWE induces a dose-dependent and chronic polyarthritis in rats. However, when injected into mice, LCWE induces instead a focal coronary arteritis ${ }^{176}$. How and which element of LCWE triggers Kawasaki disease vasculitis is unknown. LCWE is mainly composed of peptidoglycans, contains high levels of rhamnose and is resistant to lysozyme degradation ${ }^{176}$.

The cardiovascular lesions induced in mice by LCWE are histologically similar to those observed in human disease. LCWE-induced Kawasaki disease vasculitis is characterized by infiltration of inflammatory cells in the aortic root, development of necrotizing arteritis in the coronary artery followed by luminal obstruction due to LMP that can lead to complete coronary artery stenosis ${ }^{181}$, recapitulating the three pathological processes of human Kawasaki disease described above (FIG. $4 a-d)$. In children with Kawasaki disease, thrombotic occlusion of the inflamed coronary artery leads to ischaemic heart disease ${ }^{23,120}$, and similarly, occluding organizing thrombus in the coronary artery can be observed in LCWE-injected mice (FIG. 4e). Acute myocarditis and chronic scarring of the coronary arteries with the formation of stenotic fragments are also observed in LCWE-induced Kawasaki disease vasculitis (FIG. 4f), even long after the acute phase ${ }^{182}$, which is similar to the fibrotic lesions that might lead children with Kawasaki disease to develop long-term cardiovascular sequelae in adulthood ${ }^{8,9}$. MRI and echocardiography in LCWE-injected mice demonstrate the presence of electrocardiographic changes (as observed in human Kawasaki disease) and myocardial dysfunction, which are responsive to anakinra therapy ${ }^{183,184}$.

The LCWE-induced Kawasaki disease vasculitis in mice is dependent on intact TLR2 and MyD88 signalling and the subsequent release of pro-inflammatory cytokines, including IL- $1 \beta$, IL- 6 and TNF $^{10}$. Genetic depletion of the TNF receptor or pharmacological blockade of the TNF signalling pathway (with infliximab (monoclonal antibodies to TNF) or etanercept (soluble TNF receptors)) protects mice from LCWEinduced Kawasaki disease vasculitis ${ }^{132,185}$. This model is also $\mathrm{T}$ cell dependent, as $\operatorname{Rag} 1^{-1-}$ mice develop fewer cardiovascular lesions ${ }^{11}$. CD ${ }^{+} \mathrm{T}$ cells are specifically required for LCWE-induced Kawasaki disease vasculitis as treatment of LCWE-injected mice with an anti-CD8-depleting antibody prevents the development of vasculitis ${ }^{181}$. This finding correlates with human disease, in which infiltrations of $\mathrm{CD}^{+} \mathrm{T}$ cells ${ }^{135}$, and particularly $\mathrm{CD}^{+} \mathrm{T}$ cells, are detected in the CAAs ${ }^{24}$. The LCWE model has also confirmed the importance

Table 1 | Comparison of the three mouse models of Kawasaki disease

\begin{tabular}{|llll|}
\hline Characteristic & $\begin{array}{l}\text { Lactobacillus casei cell wall } \\
\text { extract }\end{array}$ & $\begin{array}{l}\text { Candida albicans water-soluble } \\
\text { fraction }\end{array}$ & Nod1 ligand (FK565) \\
\hline Induction & Single intraperitoneal injection & Repeated intraperitoneal injections & $\begin{array}{l}\text { Priming with LPS } \\
\text { and Nod1 ligand } \\
\text { intraperitoneal injection }\end{array}$ \\
\hline Pathology & $\begin{array}{l}\text { Aortic root inflammation; coronary } \\
\text { arteritis; epicardial coronary } \\
\text { arteritis; luminal myofibroblast } \\
\text { proliferation; development of } \\
\text { abdominal aorta aneurysms }\end{array}$ & $\begin{array}{l}\text { Aortic root inflammation; coronary } \\
\text { arteritis; inflammation focally } \\
\text { extending to coronary arteries; } \\
\text { development of abdominal aorta } \\
\text { aneurysms }\end{array}$ & $\begin{array}{l}\text { Aortic root inflammation; } \\
\text { coronary arteritis }\end{array}$ \\
\hline $\begin{array}{l}\text { Immune } \\
\text { characteristics }\end{array}$ & $\begin{array}{l}\text { MyD88-TLR2-dependent; NLRP3 } \\
\text { inflammasome-dependent; } \\
\text { innate immune cell dependent } \\
\text { (neutrophils and macrophages); }\end{array}$ & $\begin{array}{l}\text { Dectin-2 receptor-dependent; } \\
\text { increased antineutrophil } \\
\text { cytoplasmic antibodies; innate } \\
\text { immune cell dependent (neutrophils } \\
\text { and macrophages); cell dependent }\end{array}$ & $\begin{array}{l}\text { T cell-independent } \\
\text { macrophage-dependent; }\end{array}$ \\
\hline Thell dependent & $\begin{array}{l}\text { IVIG; anakinra; IL-1 } \alpha \text { antibody; } \\
\text { IL-1 } \beta \text { antibody; TNF antibody }\end{array}$ & $\begin{array}{l}\text { IVIG; IL-1 } \beta \text { antibody; GM-CSF } \\
\text { antibody }\end{array}$ & NA \\
\hline
\end{tabular}

GM-CSF, granulocyte-macrophage colony-stimulating factor; IVIG, intravenous immunoglobulin; LPS, lipopolysaccharide; NA, not available. 

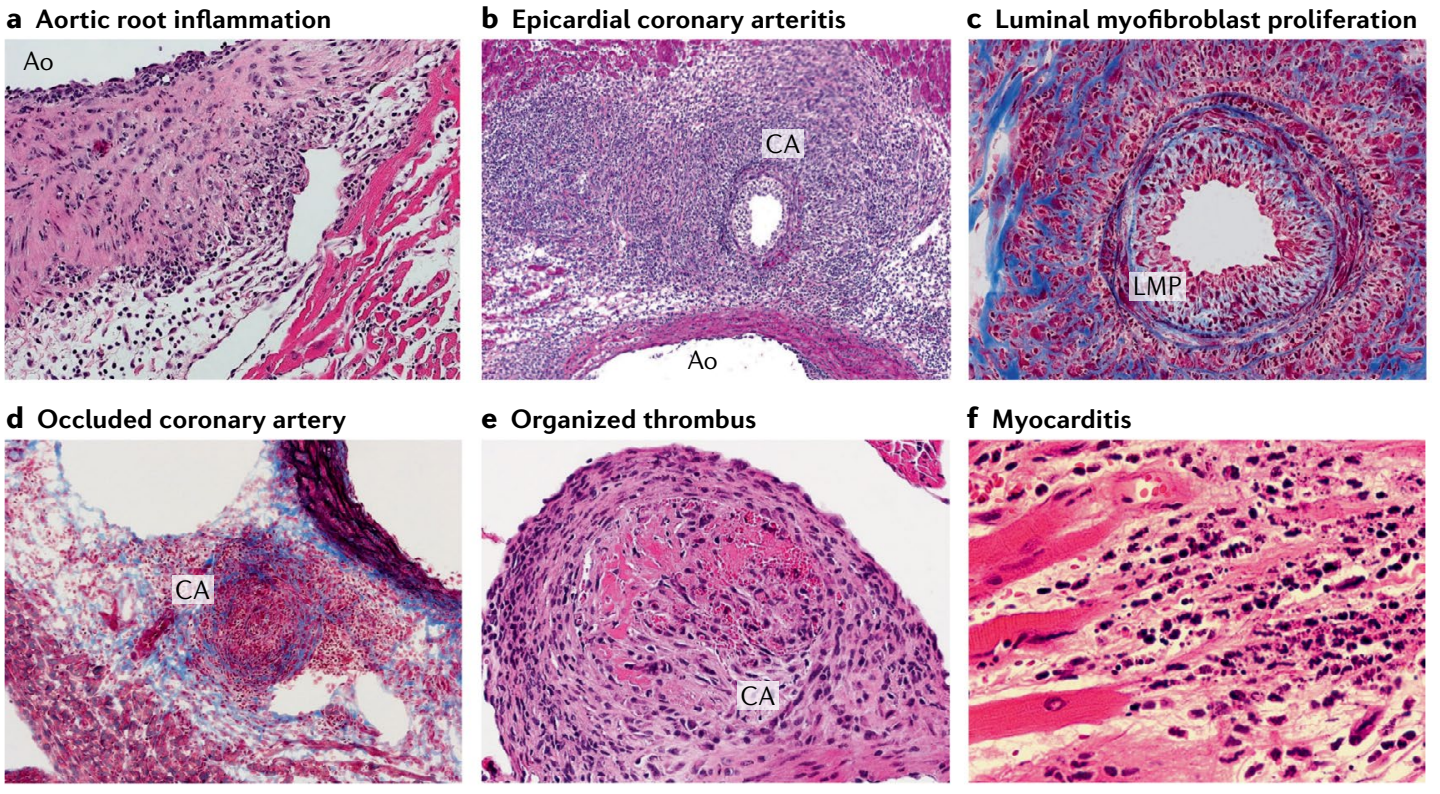

Fig. 4 | Histological and morphological findings in the LCWE-induced mouse model of Kawasaki disease vasculitis. Wild-type mice underwent intraperitoneal injection with Lactobacillus casei cell wall extract (LCWE), and heart tissues were harvested 2 weeks later. Haematoxylin and eosin (HEE) and trichrome staining were performed on heart sections. a | Inflammatory cell infiltration in the aortic route (H\&E staining; $\times 40)$. b | Arteritis development in epicardial muscular coronary artery (HEE staining; $\times 20$ ). c | Luminal myofibroblast proliferation (LMP) and non-specific neointimal proliferation injury to the arterial wall (trichrome staining; $\times 200$ ). $\mathbf{d} \mid$ Complete occlusion of the coronary artery by LMP (trichrome staining; $\times 20$ ). e $\mid$ Organized thrombus in the coronary artery (H\&E staining; $\times 200)$. f $\mid$ Myocarditis (H\&E staining; $\times 200)$. Ao; aorta, CA; coronary artery.

of the ITPKC pathway in Kawasaki disease development and demonstrated that ITPKC deficiency is associated with increased $\mathrm{Ca}^{2+}$ flux and levels of IL- $1 \beta$ in vitro ${ }^{59}$. Interestingly, the relatively mild development of coronary arteritis in LCWE-injected CBA/N mice - which are characterized by a defective $\mathrm{B}$ cell maturation process and poor humoral immune responses - suggests that the humoral immune response might participate in amplification of the disease ${ }^{186}$. $\operatorname{IgA}^{+}$plasma cells infiltrate vascular and non-vascular tissues during the acute phase of Kawasaki disease ${ }^{25,26}$, resulting in the development of an oligoclonal IgA response in the coronary $\operatorname{artery}^{125,126}$. Interestingly, we have observed increased numbers of $\operatorname{Ig} \mathrm{A}^{+}$plasmablasts in the spleen, Peyer's patches and abdominal aorta draining lymph nodes of LCWE-injected mice, as well as increased concentrations of circulating IgA and IgA deposition in heart tissues, abdominal aorta and kidney glomureli ${ }^{143}$.

Mouse models also provide a useful opportunity to evaluate the efficacy of therapeutic regimens on the development and healing of cardiovascular lesions. When given up to 5 days after LCWE injection, IVIG substantially decreases the severity of cardiovascular lesions in mice ${ }^{187}$, mirroring the effects of IVIG treatment in humans. As described above, IL- $1 \beta$ signalling is higher in patients with Kawasaki disease than in age-matched control patients with other febrile illnesses ${ }^{91,188}$, and studies using the LCWE model helped lead to the discovery of the importance of this pathway in the pathogenesis of the disease and the therapeutic potential of IL-1 blockade. Depletion of macrophages or blocking the IL-1 pathway either genetically using
$I L 1 R^{-/-}, I L 1 \alpha^{-/-}$or $I L 1 \beta^{-/-}$mice or with antibodies targeting IL- $1 \alpha$ or IL- $1 \beta$, or anakinra (IL1Ra), strongly reduces cardiovascular lesion development as well as myocardial dysfunction in LCWE-injected mice ${ }^{128,132,184}$.

The CAWS mouse model. C. albicans is a harmless commensal fungus normally present in the human gastrointestinal tract that can transition into a pathogen capable of inducing inflammation in immune-impaired hosts. In 1979, Murata demonstrated that an alkaline extract made from C. albicans isolated from faeces from a patient with Kawasaki disease induced coronary arteritis in mice $\mathrm{e}^{177}$. CAWS is composed of polysaccharides, mainly $\beta$-glucans and $\alpha$-mannan proteins of the yeast cell wall ${ }^{189}$, and needs to be injected intraperitoneally for five consecutive days in the first week of the disease to induce vasculitis in the aortic valves and the coronary arteries ${ }^{189,190}$. In this model, recognition of a-mannan proteins by the dectin- 2 receptor seems to be essential, as CAWS-injected Dectin- $2^{-/-}$mice do not develop vasculitis ${ }^{191}$.

The CAWS model shares some histological similarities with human Kawasaki disease pathology in that inflammation affects both the aortic root and the proximal region of the coronary arteries ${ }^{190}$. Inflammation can also affect non-coronary artery sites in $25 \%$ of CAWS-injected mice and can be observed in the lymph nodes, the kidneys and the liver ${ }^{190,192}$. CAWS-induced coronary artery lesions resemble those of human Kawasaki disease and are typically proliferative, granulomatous and characterized by intimal thickening with destruction of the elastic lamina and media ${ }^{190}$. 
Echocardiography in CAWS-injected mice indicates a marked decrease of cardiac function, which can be restored by IL-10 supplementation ${ }^{193}$. IL-10 is a potent anti-inflammatory cytokine that might improve the outcome of CAWS-induced vasculitis by inhibiting the release of pro-inflammatory mediators, such as TNF and IL- $1 \beta$, from tissue-infiltrating innate immune cells ${ }^{194}$. Interestingly, CAWS-induced Kawasaki disease vasculitis is also strain dependent, as CAWS injections lead to a high incidence of vasculitis in $\mathrm{CD}-1, \mathrm{C} 3 \mathrm{H} / \mathrm{HeN}$, $\mathrm{DBA} / 2$ and $\mathrm{C} 57 \mathrm{BL} / 6 \mathrm{~N}$ mice, but the $\mathrm{CBA} / \mathrm{JN}$ strain is resistant to coronary arteritis ${ }^{190,195}$. The DBA/2 strain is the most sensitive, with the highest mortality rate resulting from a more intense coronary arteritis ${ }^{195}$. The sensitivity of DBA/2 mice is associated with increased production of the pro-inflammatory cytokines TNF, IL- 6 and IFN $\gamma^{195,196}$, whereas resistance of CBA/JN mice is explained by increased levels of IL-10 production in that strain ${ }^{197}$.

Despite the presence of $\mathrm{T}$ cell and $\mathrm{B}$ cell infiltration in the inflamed coronary artery, mice lacking $\mathrm{T}$ cells still develop moderate to typical cardiac inflammation, indicating that $\mathrm{T}$ cells might not be required in the development of Kawasaki disease vasculitis in this particular model ${ }^{198,199}$. Absence of both T cells and B cells in Rag $^{-1-}$ mice leads to lower incidence of CAWS-induced Kawasaki disease vasculitis; reconstitution of $\mathrm{Rag1}^{-1-}$ mice with wild-type, but not $C C R 2^{-/-}$, T cells and $\mathrm{B}$ cells restores cardiovascular lesions, suggesting roles for both $\mathrm{T}$ cells and $\mathrm{B}$ cells and the modulation of disease development by CCR 2 expression ${ }^{200}$. The innate immune response also participates in vasculitis development; resident macrophages recognize the CAWS antigens through the dectin-2 receptor, leading to their activation, release of CCL2, and recruitment of neutrophils and inflammatory monocytes producing IL- $1 \beta$ in the aortic $\operatorname{root}^{201}$.

CAWS-induced vasculitis is also associated with the rapid production of granulocyte-monocyte colonystimulating factor in the heart, which subsequently drives inflammatory myocarditis by activating tissue macrophages and promoting recruitment of neutrophils and monocytes ${ }^{199}$. TNF is also produced during the acute phase of CAWS-induced Kawasaki disease vasculitis and is essential for the development of acute myocarditis, as TNF receptor-deficient mice are protected from the development of CAWS vasculitis ${ }^{202}$. IVIG administration substantially reduces CAWS-induced heart vessel inflammation ${ }^{203}$. Like the LCWE model, the CAWS model is also dependent on the IL-1 pathway, as $I L 1 R^{-/-}, I L 1 \beta^{-/-}, A s c^{-/-}$and $N \operatorname{lrp} 3^{-/-}$mice are protected from induction of vasculitis, and treatment with anti-IL- $1 \beta$ agents substantially attenuates CAWS vasculitis $202,204,205$.

The Nod1 ligand mouse model. Endothelial cells are equipped to sense microbial components through Toll-like receptors and nucleotide-binding oligomerization domain-containing protein like receptors. Subcutaneous injection or oral delivery of FK565, a specific synthetic Nod1 ligand, in mice primed with LPS results in a diffuse cellular inflammation of the aortic root and transmural infiltration of inflammatory cells in the coronary artery wall ${ }^{178,206}$. Other arteries, such as the iliac and renal arteries, also show signs of inflammation associated with a thickening of the intima ${ }^{206}$.

The mechanisms by which FK565 induces coronary arteritis in mice remain unknown. When administered orally, FK565 does not induce intestinal mucosa inflammation, but specifically activates vascular cells to produce a diverse array of pro-inflammatory cytokines, including IL- $1 \beta^{206}$, and chemokines such as CCL2, resulting in the recruitment of inflammatory cells in the tissues ${ }^{178}$. This model seems to be independent of $\mathrm{T}$ cells, B cells and natural killer T cells, as LPS-primed Rag- $1^{-/-}$mice still develop aortitis and coronary arteritis after FK565 injection ${ }^{207}$. The inflammatory infiltrates observed around the inflamed aortic root and coronary arteries mainly comprise neutrophils and CD $11 \mathrm{c}^{+}$ cardiac macrophages; their specific depletion considerably reduces the development of FK565-induced Kawasaki disease vasculitis ${ }^{178,207}$. The concentration of circulating IL- $1 \beta$ is substantially increased in the serum of FK565-injected mice compared with control or CAWS-injected animals, and higher IL-1 $\beta$ levels correlate with a larger inflammation area ${ }^{206}$. However, specific studies further investigating the role of IL- $1 \beta$ in this model are needed.

\section{Treatment of Kawasaki disease}

Traditional and novel therapies in humans. The current standard of care for Kawasaki disease is the use of high-dose IVIG together with aspirin. If given during the first 10 days of the disease, IVIG reduces the risk of development of coronary arteritis and aneurysms from about $30 \%$ to $5-7 \%{ }^{14,15}$. The mechanisms by which IVIG treatment reduces the inflammatory responses are still unknown; however, IVIG is suspected to have a wide spectrum of action targeting multiple arms of the immune response ${ }^{18}$. IVIG has been shown to inhibit IL- $1 \beta$ production from in vitro stimulated macrophages and to stimulate the production of IL-1 $\mathrm{Ra}^{208,209}$. During Kawasaki disease, IVIG reduces production of inflammatory cytokines and chemokines, and decreases the activation and number of circulating neutrophils, monocytes, macrophages and activated $\mathrm{T}$ cells by saturating Fc receptors ${ }^{18}$. The majority of patients with Kawasaki disease who are treated with IVIG improve and do not develop coronary artery damage; however, up to $20 \%$ of children with Kawasaki disease do not respond to treatment or have fever recurrence after initial IVIG treatment, and these patients are at the highest risk of developing coronary artery lesions $\mathrm{s}^{3,20,210}$.

The involvement of pro-inflammatory cytokines in the acute phase of Kawasaki disease suggests that combinational therapy, composed of IVIG associated with TNF inhibitors, steroids, calcineurin inhibitors or anakinra, might be useful to treat patients with IVIG-resistant disease. The use of TNF inhibitors in combination with IVIG has had mixed results thus far. Infliximab was associated with decreased fever duration and reduced markers of inflammation (C-reactive protein and neutrophil counts), suggesting a possible improvement of coronary artery outcomes ${ }^{211}$; however, etanercept treatment 
resulted in a substantial reduction in IVIG resistance only in patients $>1$ year old ${ }^{212}$.

An important area of research is the use of biomarkers to predict IVIG resistance in Kawasaki disease. The Kobayashi scoring system, based on a combination of laboratory test results (for example, C-reactive protein levels, neutrophil percentages, platelets counts and levels of aspartate and alanine aminotransferase) and demographic variables (sex, age and number of days of illness before the start of the treatment) has been successfully used to predict IVIG-resistance in Japanese patients ${ }^{213}$, but not in North American children with Kawasaki disease $^{214}$. The combination of prednisolone and IVIG to treat Japanese patients with Kawasaki disease predicted to have IVIG-resistant disease according to the Kobayashi score (RAISE study) resulted in more rapid fever resolution, reduced development of CAAs and lower incidence of additional rescue treatment ${ }^{215}$ compared with IVIG alone.

As discussed above, Kawasaki disease susceptibility and increased coronary artery lesion risk are associated with an SNP in ITPKC ${ }^{58}$ that results in a lack of NFAT regulation and activation of the $\mathrm{T}$ cell compartment owing to increased IL-2 production ${ }^{216}$. CD8 ${ }^{+}$cytotoxic $\mathrm{T}$ cells are present in the inflamed arterial wall during Kawasaki disease $\mathrm{e}^{24,135}$; therefore, targeting T cell expansion might be an efficient approach to preventing CAAs during Kawasaki disease. A combination treatment of IVIG and ciclosporin, a calcineurin inhibitor that suppresses IL-2 production and T cell activation, was tested in a clinical trial in Japanese patients with Kawasaki disease predicted to have IVIG-resistant disease based on the Kobayashi score (KAICA trial) ${ }^{217}$. In this trial, the combination treatment was shown to be safe and associated with a lower incidence of CAAs; however, treatment was linked with increased risk of relapse ${ }^{217}$. Furthermore, the scoring system used to identify IVIG-non-responders is poorly predictive in European children with Kawasaki disease, limiting the conclusions of this study.

The important role of the IL-1 $\beta$-IL- 1 receptor pathway in Kawasaki disease development has been demonstrated in both human patients $\mathrm{s}^{27,28,129,130}$ and mouse models ${ }^{127,132,202,204}$. Therefore, clinical trials investigating IL-1 pathway inhibition by using anakinra, which blocks both IL- $1 \alpha$ and IL- $1 \beta$, have been initiated in North America (ANAKID; ClinicalTrials.gov identifier NCT02179853) ${ }^{218}$ and Europe (Kawakinra; European Clinical Trials number 2014-002715-4 $)^{219}$. Already, multiple case reports exist of the successful use of anakinra to treat patients with IVIG-resistant Kawasaki disease ${ }^{220-224}$, indicating the promise of this second-line therapy.

Therapeutic insights from mouse models. Although no animal model can fully mimic human disease, the LCWE-induced Kawasaki disease mouse model has been accepted by many in the research community as a reliable experimental model providing novel insights that can be tested in patients. For example, IVIG efficiently prevents coronary arteritis development in LCWE-injected mice ${ }^{187}$ as well as in the CAWS mouse model of Kawasaki disease ${ }^{203}$.
The effects of the calcineurin inhibitors ciclosporin and tacrolimus have been investigated in the Nod1 ligand-induced mouse model of Kawasaki disease vasculitis ${ }^{225}$. This approach was rational given the established role of $\mathrm{T}$ cells and calcium signalling in Kawasaki disease. However, contrary to the expected outcome, these inhibitors exacerbated the coronary $\operatorname{arteritis}^{225}$. Notably, however, this result was probably related to the choice of mouse model, as the Nod1 ligand-mediated mouse model of Kawasaki disease vasculitis has previously been shown to be T cellindependent ${ }^{207}$. Indeed, in an independent study using the CAWS mouse model, which is T cell dependent, ciclosporin suppressed CAWS-induced vasculitis ${ }^{226}$, emphasizing the importance of model selection in preclinical studies. Most importantly, results in human studies bear out the therapeutic potential of calcineurin inhibition, as the Japanese phase III trial (KAICA trial) showed that adding ciclosporin to IVIG in patients with Kawasaki disease who were at high risk of IVIG resistance was beneficial in diminishing overall incidence of CAAs ${ }^{217}$.

The role of TNF has been investigated in both the LCWE and the CAWS mouse models of Kawasaki disease vasculitis ${ }^{185}$. Initially, etanercept treatment or genetic deletion of TNF receptor 1 was shown to protect mice from LCWE-induced coronary arteritis ${ }^{185,202}$. Infliximab treatment also prevented the development of both LCWE-induced coronary arteritis and myocarditis $^{132}$. Similar results were obtained in the CAWS mouse model of Kawasaki disease vasculitis, in which etanercept ${ }^{226,227}$ suppressed the incidence and decreased the severity of vasculitis. Mechanistically, TNF has been proposed to be produced by myeloid cells in the acute phase and to promote myocarditis and recruitment of immune cells by acting on cardiac stromal cells $^{202}$. However, infliximab and etanercept might not directly target the TNF signalling pathway, and their observed effects might be indirect. Indeed, infliximab is not able to bind mouse $\mathrm{TNF}^{227,228}$; therefore, the anti-inflammatory effect of infliximab might be attributable to the binding of $\mathrm{Fc}$ receptors at the surface of activated cells ${ }^{229,230}$.

The overwhelming evidence for the critical role of IL- $1 \beta$ in promoting LCWE-induced Kawasaki disease vasculitis in mice ${ }^{127,128,132}$ led to the initiation of clinical trials testing the effect of anakinra for blocking IL- $1 \beta$ as a second therapy option to treat children with IVIG-resistant Kawasaki disease. Multiple case reports now outline the successful use of anakinra to treat patients with IVIG-resistant Kawasaki disease ${ }^{221-224}$. Alternatively, direct inhibition of the NLRP3 inflammasome might be a more targeted therapeutic strategy to treat Kawasaki disease, as it would affect several pathways beyond IL- $1 \beta$, including IL- $1 \alpha$ and IL- 18 . Several NLRP3 inhibitors have been identified ${ }^{231}$ and tested in mouse models of inflammatory diseases, such as experimental autoimmune encephalomyelitis and cryopyrin-associated periodic syndrome ${ }^{232}$. It would be interesting to determine if such drugs could be used to prevent and reduce the cardiovascular complications in mouse models of Kawasaki disease vasculitis. 


\section{Conclusions}

Over the past 40 years, research has improved our understanding of Kawasaki disease pathology and the development of coronary vasculitis. However, some questions still remain unanswered, such as the identification of the aetiological agents, how the disease is triggered, and the specific immune pathways associated with coronary vasculitis development and IVIG resistance. Owing to the rarity of human tissues from patients with Kawasaki disease, the use of animal models reproducing human Kawasaki disease features is invaluable. Many advances have been made over the decades by combining biological observations in human samples with mechanistic insights from experimental animal models. This 'bench to bedside' approach successfully led to the identification of the critical role of IL- $1 \beta$ in Kawasaki disease and resulted in the development of clinical trials in which anakinra is being used to treat children with IVIG-resistant Kawasaki disease.

LCWE-injected mice exhibit a dysfunctional intestinal barrier, and the increased IgA response and elevated sIgA levels in both LCWE-injected mice and children with Kawasaki disease reveal the existence of a 'gutvascular' axis $^{143}$. In evaluating this model system and the role of $\operatorname{IgA}$, it should not be forgotten that injection of identically prepared LCWE induces chronic polyarthritis in selected inbred rat strains ${ }^{180,233}$. This observation implies that a common immunogenetic pathway might underlie a variety of autoimmune illnesses, with disease expression moderated not by the inducing agent, but rather by host genetics. The fact that cell wall fragments of common gut bacteria can produce varying disease manifestations in the face of inflammation-induced increased gut permeability suggests that some autoimmune diseases might not in fact be induced by the normal response to an unusual agent, but rather an unusual response to a common agent. Similarly, we hypothesize that vasculitic diseases, including Kawasaki disease, are not a usual response to an unusual environmental stimulus, but rather an unusual response (genetically determined) to a common environmental stimulus. This hypothesis has major implications for understanding the aetiology and pathogenesis of not only Kawasaki disease but also IgA-mediated diseases and perhaps others. In addition, it strongly suggests that inhibition of IL- $1 \beta$ might be effective for the many chronic inflammatory diseases in which IgA deposition is a key finding.

Published online 26 May 2020
1. Kawasaki, T., Kosaki, F., Okawa, S., Shigematsu, I. \& Yanagawa, H. A new infantile acute febrile mucocutaneous lymph node syndrome (MLNS) prevailing in Japan. Pediatrics 54, 271-276 (1974)

2. Newburger, J. W., Takahashi, M. \& Burns, J. C. Kawasaki disease. J. Am. Coll. Cardiol. 67, 1738-1749 (2016).

3. McCrindle, B. W. et al. Diagnosis, treatment, and long-term management of Kawasaki disease: a scientific statement for health professionals from the American Heart Association. Circulation 135 e927-e999 (2017).

4. Newburger, J. W. et al. Diagnosis, treatment, and long-term management of Kawasaki disease: a statement for health professionals from the Committee on Rheumatic Fever, Endocarditis and Kawasaki Disease, Council on Cardiovascular Disease in the Young, American Heart Association. Circulation 110, 2747-2771 (2004).

5. Singh, S., Vignesh, P. \& Burgner, D. The epidemiology of Kawasaki disease: a global update. Arch. Dis. Child. 100, 1084-1088 (2015).

6. Gordon, J. B., Kahn, A. M. \& Burns, J. C. When children with Kawasaki disease grow up: myocardial and vascular complications in adulthood. J. Am. Coll. Cardiol. 54, 1911-1920 (2009).

7. Daniels, L. B. et al. Prevalence of Kawasaki disease in young adults with suspected myocardial ischemia. Circulation 125, 2447-2453 (2012).

8. Rizk, S. R. et al. Acute myocardial ischemia in adults secondary to missed Kawasaki disease in childhood. Am. J. Cardiol. 115, 423-427 (2015).

9. Gordon, J. B. et al. The spectrum of cardiovascular lesions requiring intervention in adults after Kawasaki disease. JACC Cardiovasc. Interv. 9, 687-696 (2016).

10. Rosenkranz, M. E. et al. TLR2 and MyD88 contribute to Lactobacillus casei extract-induced focal coronary arteritis in a mouse model of Kawasaki disease. Circulation 112, 2966-2973 (2005).

11. Schulte, D. J. et al. Involvement of innate and adaptive immunity in a murine model of coronary arteritis mimicking Kawasaki disease. J. Immunol. 183 5311-5318 (2009).

12. Rowley, A. H., Baker, S. C., Orenstein, J. M. $\delta$ Shulman, S. T. Searching for the cause of Kawasak disease-cytoplasmic inclusion bodies provide new insight Nat. Rev Microbiol. 6, 394-401 (2008).

13. Rowley, A. H. et al. Ultrastructural, immunofluorescence, and RNA evidence support the hypothesis of a new virus associated with Kawasaki disease. J. Infect. Dis. 203, 1021-1030 (2011)
14. Burns, J. C., Capparelli, E. V., Brown, J. A., Newburger, J. W. \& Glode, M. P. Intravenous gammaglobulin treatment and retreatment in Kawasaki disease. US/Canadian Kawasaki Syndrome Study Group. Pediatr. Infect. Dis. J. 17, 1144-1148 (1998).

15. Tremoulet, A. H. et al. Resistance to intravenous immunoglobulin in children with Kawasaki disease. J. Pediatr. 153, 117-121 (2008)

16. Kato, $\mathrm{H}$. et al. Long-term consequences of Kawasak disease. A 10- to 21-year follow-up study of 594 patients. Circulation 94, 1379-1385 (1996).

17. Friedman, K. G. et al. Coronary artery aneurysms in Kawasaki disease: risk factors for progressive disease and adverse cardiac events in the US population. J. Am. Heart Assoc. 5, e003289 (2016).

18. Burns, J. C. \& Franco, A. The immunomodulatory effects of intravenous immunoglobulin therapy in Kawasaki disease. Expert. Rev. Clin. Immunol. 11 , 819-825 (2015)

19. Moffett, B. S., Syblik, D., Denfield, S., Altman, C. \& Tejtel-Sexson, K. Epidemiology of immunoglobulin resistant Kawasaki disease: results from a large, national database. Pediatr. Cardiol. 36, 374-378 (2015).

20. Skochko, S. M. et al. Kawasaki disease outcomes and response to therapy in a multiethnic community: a 10-year experience. J. Pediatr. 203, 408-415 (2018).

21. Makino, N. et al. Nationwide epidemiologic survey of Kawasaki disease in Japan, 2015-2016. Pediatr. Int. 61, 397-403 (2019).

22. Takahashi, K., Oharaseki, T., Yokouchi, Y., Hiruta, N. $\&$ Naoe, S. Kawasaki disease as a systemic vasculitis in childhood. Ann. Vasc. Dis. 3, 173-181 (2010).

23. Takahashi, K., Oharaseki, T. \& Yokouchi, Y. Histopathological aspects of cardiovascular lesions in Kawasaki disease. Int. J. Rheum. Dis. 21, 31-35 (2017).

24. Brown, T. J. et al. CD8 T lymphocytes and macrophages infiltrate coronary artery aneurysms in acute Kawasaki disease. J. Infect. Dis. 184, 940-943 (2001).

25. Rowley, A. H., Eckerley, C. A., Jack, H. M., Shulman, S. T. $\S$ Baker, S. C. IgA plasma cells in vascular tissue of patients with Kawasaki syndrome. J. Immunol. 159 5946-5955 (1997).

26. Rowley, A. H. et al. IgA plasma cell infiltration of proximal respiratory tract, pancreas, kidney, and coronary artery in acute Kawasaki disease. J. Infect. Dis. 182, 1183-1191 (2000).

27. Leung, D. Y. et al. Two monokines, interleukin 1 and tumor necrosis factor, render cultured vascular endothelial cells susceptible to lysis by antibodies circulating during Kawasaki syndrome J. Exp. Med. 164, 1958-1972 (1986).

28. Leung, D. Y. et al. Endothelial cell activation and high interleukin-1 secretion in the pathogenesis of acute Kawasaki disease. Lancet 2, 1298-1302 (1989).

29. Rowley, A. H. Is Kawasaki disease an infectious disorder? Int. J. Rheum. Dis. 21, 20-25 (2018).

30. Uehara, R. \& Belay, E. D. Epidemiology of Kawasaki disease in Asia, Europe, and the United States. J. Epidemiol. 22, 79-85 (2012).

31. Holman, R. C. et al. Hospitalizations for Kawasaki syndrome among children in the United States, 1997-2007. Pediatr. Infect. Dis. J. 29, 483-488 (2010).

32. Faulkner, L., Cooper, A., Fantino, C., Altmann, D. M. \& Sriskandan, S. The mechanism of superantigenmediated toxic shock: not a simple Th 1 cytokine storm. J. Immunol. 175, 6870-6877 (2005).

33. Abe, J. et al. Selective expansion of $\mathrm{T}$ cells expressing T-cell receptor variable regions $\mathrm{V}$ beta 2 and $\mathrm{V}$ beta 8 in Kawasaki disease. Proc. Natl Acad. Sci. USA 89 , 4066-4070 (1992).

34. Abe, J. et al. Characterization of T cell repertoire changes in acute Kawasaki disease. J. Exp. Med. 177 791-796 (1993).

35. Curtis, N., Zheng, R., Lamb, J. R. \& Levin, M Evidence for a superantigen mediated process in Kawasaki disease. Arch. Dis. Child. 72, 308-311 (1995).

36. Mancia, L. et al. Characterization of the T-cell receptor V-beta repertoire in Kawasaki disease. Scand. J. Immunol. 48, 443-449 (1998).

37. Pietra, B. A., De Inocencio, J., Giannini, E. H. $\&$ Hirsch, R. TCR V beta family repertoire and T cell activation markers in Kawasaki disease. J. Immunol. 153, 1881-1888 (1994)

38. Iwanaga, M. et al. Kawasaki disease and Epstein-Barr virus. Lancet 317, 938-939 (1981).

39. Kikuta, H. et al. Recurrence of Kawasaki disease and Epstein-Barr virus infection. J. Infect. Dis. 162 1215-1215 (1990).

40. Kikuta, $\mathrm{H}$ et al. Epstein-Barr virus genome-positive T lymphocytes in a boy with chronic active EBV infection associated with Kawasaki-like disease. Nature 333, 455-457 (1988).

41. Marchette, N. J. et al. Epstein-Barr virus and other herpesvirus infections in Kawasaki syndrome. J. Infect. Dis. 161, 680-684 (1990).

42. Fuse, S. et al. Children with Kawasaki disease are not infected with Epstein-Barr virus. Pediatr. Infect. Dis. J. 29, 286-287 (2010). 
43. Lee, S. J., Lee, K. Y., Han, J. W., Lee, J. S. \& Whang, K. T Epstein-Barr virus antibodies in Kawasaki disease Yonsei Med. J. 47, 475-479 (2006)

44. Esper, F. et al. Association between a novel human coronavirus and Kawasaki disease. J. Infect. Dis. 191, 499-502 (2005)

45. Ebihara, T., Endo, R., Ma, X., Ishiguro, N. \& Kikuta, H. Lack of association between New Haven coronavirus and Kawasaki disease. J. Infect. Dis. 192, 351-352 (2005).

46. Belay, E. D. et al. Kawasaki disease and human coronavirus. J. Infect. Dis. 192, 352-353; author reply 353 (2005)

47. Shulman, S. T. \& Rowley, A. H. Does Kawasaki disease have a retroviral aetiology? Lancet 2, 545-546 (1986).

48. Burns, J. C. et al. Polymerase activity in lymphocyte culture supernatants from patients with Kawasaki disease. Nature 323, 814-816 (1986).

49. Nigro, G. \& Midulla, M. Retrovirus and Kawasaki disease. Lancet 2, 1045 (1986).

50. Rauch, A. M., Fultz, P. N. \& Kalyanaraman, V. S. Retrovirus serology and Kawasaki syndrome. Lancet 329, 1431 (1987).

51. Okamoto, T. et al. Lack of evidence of retroviral involvement in Kawasaki disease. Pediatrics 81, 599 (1988).

52. Rowley, A. H. et al. A protein epitope targeted by the antibody response to Kawasaki disease. J. Infect. Dis. https://doi.org/10.1093/infdis/jiaa066 (2020).

53. Rowley, A. H. et al. Monoclonal antibodies from children with Kawasaki disease (KD) recognize hepacivirus peptides [abstract]. Presented at the 2019 Pediatric Academic Societies Meeting https://www.xcdsystem.com/pas2019/program/2019/ index. cfm?pgid $=156 \&$ sid $=1060$ (2019)

54. Rodo, X. et al. Tropospheric winds from northeastern China carry the etiologic agent of Kawasaki disease from its source to Japan. Proc. Natl Acad. Sci. USA 111, 7952-7957 (2014).

55. Fujita, Y et al. Kawasaki disease in families. Pediatrics 84, 666-669 (1989).

56. Jayaraman, T., Ondriasova, E., Ondrias, K., Harnick, D. J. \& Marks, A. R. The inositol 1,4,5-trisphosphate receptor is essential for T-cell receptor signaling. Proc. Natl Acad. Sci. USA 92, 6007-6011 (1995)

57. Kuo, H. C. et al. ITPKC single nucleotide polymorphism associated with the Kawasaki disease in a Taiwanese population. PLoS One 6, e17370 (2011).

58. Onouchi, Y. et al. ITPKC functional polymorphism associated with Kawasaki disease susceptibility and formation of coronary artery aneurysms. Nat. Genet 40, 35-42 (2008).

59. Alphonse, M. P. et al. Inositol-triphosphate 3-kinase C mediates inflammasome activation and treatment response in Kawasaki disease. J. Immunol. 197 3481-3489 (2016).

60. Kuo, H. C. et al. Lack of association between ORAI1/CRACM 1 gene polymorphisms and Kawasak disease in the Taiwanese children. J. Clin. Immunol. 31, 650-655 (2011).

61. Onouchi, Y. et al. Variations in ORAl1 gene associated with Kawasaki disease. PLoS One 11, e0145486 (2016).

62. Shimizu, C. et al. Genetic variation in the SLC8A1 calcium signaling pathway is associated with susceptibility to Kawasaki disease and coronary artery abnormalities. Circ. Cardiovasc. Genet. 9, 559-568 (2016)

63. Murakami, T. et al. Critical role for calcium mobilization in activation of the NLRP3 inflammasome. Proc. Nat Acad. Sci. USA 109, 11282-11287 (2012).

64. Rossol, M. et al. Extracellular $\mathrm{Ca} 2+$ is a danger signal activating the NLRP3 inflammasome through $G$ protein-coupled calcium sensing receptors. Nat. Commun. 3, 1329 (2012).

65. Elgueta, R. et al. Molecular mechanism and function of $C D 40 / C D 40 L$ engagement in the immune system. Immunol. Rev. 229, 152-172 (2009).

66. Wang, C. L. et al. Expression of CD40 ligand on CD4+ T-cells and platelets correlated to the coronary artery lesion and disease progress in Kawasaki disease. Pediatrics 111, E140-E147 (2003).

67. Onouchi, Y. et al. CD40 ligand gene and Kawasaki disease. Eur. J. Hum. Genet. 12, 1062-1068 (2004).

68. Huang, F. Y. et al. Genetic polymorphisms in the CD40 ligand gene and Kawasaki disease. J. Clin. Immunol. 28, 405-410 (2008)

69. Kuo, H.-C. et al. CD40 gene polymorphisms associated with susceptibility and coronary artery lesions of
Kawasaki disease in the Taiwanese population. ScientificWorldJournal 2012, 520865 (2012)

70. Turner, M. W. The role of mannose-binding lectin in health and disease. Mol. Immunol. 40, 423-429 (2003).

71. Biezeveld, M. H. et al. Polymorphisms in the mannose-binding lectin gene as determinants of age-defined risk of coronary artery lesions in Kawasaki disease. Arthritis Rheum. 54, 369-376 (2006)

72. Biezeveld, M. H. et al. Association of mannose-binding lectin genotype with cardiovascular abnormalities in Kawasaki disease. Lancet 361, 1268-1270 (2003).

73. Sato, S. et al. Association of mannose-binding lectin gene polymorphisms with Kawasaki disease in the Japanese. Int. J. Rheum. Dis. 12, 307-310 (2009).

74. Nakamura, A. et al. Involvement of mannose-binding lectin in the pathogenesis of Kawasaki disease-like murine vasculitis. Clin. Immunol. 153, 64-72 (2014).

75. Manger, K. et al. Fcgamma receptor Ila polymorphism in Caucasian patients with systemic lupus erythematosus: association with clinical symptoms. Arthritis Rheum. 41, 1181-1189 (1998).

76. Brun, J. G., Madland, T. M. \& Vedeler, C. A Immunoglobulin G fc-receptor (FcgammaR) IIA IIIA, and IIIB polymorphisms related to disease severity in rheumatoid arthritis. J. Rheumatol. 29 1135-1140 (2002)

77. Bredius, R. G. et al. Fc gamma receptor Ila (CD32) polymorphism in fulminant meningococcal septic shock in children. J. Infect. Dis. 170, 848-853 (1994).

78. Biezeveld, M. et al. The involvement of Fc gamma receptor gene polymorphisms in Kawasaki disease. Clin. Exp. Immunol. 147, 106-111 (2007).

79. Khor, C. C. et al. Genome-wide association study identifies FCGR2A as a susceptibility locus for Kawasaki disease. Nat. Genet. 43, 1241-1246 (2011).

80. Tremoulet, A. H. Adjunctive therapies in Kawasaki disease. Int. J. Rheum. Dis. 21, 76-79 (2018).

81. Orenstein, J. M. et al. Three linked vasculopathic processes characterize Kawasaki disease: a light and transmission electron microscopic study. PLoS One 7 , e38998 (2012).

82. Austermann, J., Spiekermann, C. \& Roth, J. S100 proteins in rheumatic diseases. Nat. Rev. Rheumatol. 14, 528-541 (2018)

83. Abe, J. et al. Gene expression profiling of the effect of high-dose intravenous Ig in patients with Kawasaki disease. J. Immunol. 174, 5837-5845 (2005).

84. Lech, M. et al. Circulating markers of inflammation persist in children and adults with giant aneurysms after Kawasaki disease. Circ. Genom. Precis. Med. 12 e002433 (2019).

85. Armaroli, G. et al. Monocyte-derived interleukin-1 $\beta$ as the driver of $\mathrm{S} 100 \mathrm{~A} 12$-induced sterile inflammatory activation of human coronary artery endothelial cells: implications for the pathogenesis of Kawasaki disease. Arthritis Rheumatol. 71, 792-804 (2019).

86. Siefert, S. A. \& Sarkar, R. Matrix metalloproteinases in vascular physiology and disease. Vascular 20 , 210-216 (2012)

87. Senzaki, H. The pathophysiology of coronary artery aneurysms in Kawasaki disease: role of matrix metalloproteinases. Arch. Dis. Child. 91, 847-851 (2006).

88. Shimizu, C. et al. Matrix metalloproteinase haplotypes associated with coronary artery aneurysm formation in patients with Kawasaki disease. J. Hum. Genet. 55, 779-784 (2010)

89. Korematsu, S. et al. Cell distribution differences of matrix metalloproteinase- 9 and tissue inhibitor of matrix metalloproteinase- 1 in patients with Kawasaki disease. Pediatr. Infect. Dis. J. 31, 973-974 (2012).

90. Johnson, J. L., Dwivedi, A., Somerville, M., George, S. J. \& Newby, A. C. Matrix metalloproteinase (MMP)-3 activates MMP-9 mediated vascular smooth muscle cell migration and neointima formation in mice. Arterioscler. Thromb. Vasc. Biol. 31, e35-e44 (2011).

91. Popper, S. J. et al. Gene-expression patterns reveal underlying biological processes in Kawasaki disease Genome Biol. 8, R261 (2007).

92. Matsuyama, T. Tissue inhibitor of metalloproteinases-1 and matrix metalloproteinase-3 in Japanese healthy children and in Kawasaki disease and their clinical usefulness in juvenile rheumatoid arthritis. Pediatr. Int. 41, 239-245 (1999).

93. Bonin, P. D., Fici, G. J. \& Singh, J. P. Interleukin-1 promotes proliferation of vascular smooth muscle cells in coordination with PDGF or a monocyte derived growth factor. Exp. Cell Res. 181, 475-482 (1989).

94. Sasu, S. \& Beasley, D. Essential roles of $1 \kappa B$ kinases $\alpha$ and $\beta$ in serum- and IL-1-induced human VSMC proliferation. Am. J. Physiol. Heart Circ. Physiol. 278 H1823-H1831 (2000).

95. Alexander, M. R. et al. Genetic inactivation of IL-1 signaling enhances atherosclerotic plaque instability and reduces outward vessel remodeling in advanced atherosclerosis in mice. J. Clin. Invest. 122, 70-79 (2012).

96. Lau, A. C., Duong, T. T., Ito, S. \& Yeung, R. S Matrix metalloproteinase 9 activity leads to elastin breakdown in an animal model of Kawasaki disease Arthritis Rheum. 58, 854-863 (2008).

97. Lau, A. C., Duong, T. T., Ito, S., Wilson, G. J. \& Yeung, R. S. Inhibition of matrix metalloproteinase-9 activity improves coronary outcome in an animal model of Kawasaki disease. Clin. Exp. Immunol. 157, 300-309 (2009).

98. Tahamtan, A., Teymoori-Rad, M., Nakstad, B. ¿ Salimi, V. Anti-inflammatory microRNAs and their potential for inflammatory diseases treatment. Front. Immunol. 9, 1377 (2018).

99. van Rooij, E. The art of microRNA research. Circ. Res. 108, 219-234 (2011).

100. Chu, M. et al. Bone marrow-derived microRNA-223 works as an endocrine genetic signal in vascular endothelial cells and participates in vascular injury from Kawasaki disease. J. Am. Heart Assoc. 6 , e004878 (2017).

101. Kuo, H.-C. et al. Next-generation sequencing identifies micro-RNA-based biomarker panel for Kawasaki disease. J. Allergy Clin. Immunol. 138, 1227-1230 (2016).

102. Rowley, A. H. et al. A study of cardiovascular miRNA biomarkers for Kawasaki disease. Pediatr. Infect. Dis. J. 33, 1296-1299 (2014)

103. Shimizu, C. et al. Differential expression of miR-145 in children with Kawasaki disease. PLoS One 8, e58159 (2013).

104. He, M et al. miR-483 targeting of CTGF suppresses endothelial-to-mesenchymal transition: therapeutic implications in Kawasaki disease. Circ. Res. 120, 354-365 (2017)

105. Rangrez, A. Y., Massy, Z. A., Meuth, V. M.-L. \& Metzinger, L. miR-143 and miR-145: molecular keys to switch the phenotype of vascular smooth muscle cells. Circ. Cardiovasc. Genet. 4, 197-205 (2011).

106. Parmacek, M. S. MicroRNA-modulated targeting of vascular smooth muscle cells. J. Clin. Invest. 119 2526-2528 (2009).

107. $\mathrm{Si}, \mathrm{X}$. et al. miR-23a downregulation modulates the inflammatory response by targeting ATG 12 -mediated autophagy. Mol. Med. Rep. 18, 1524-1530 (2018).

108. Long, B., Gan, T.-Y., Zhang, R.-C. \& Zhang, Y.-H. miR-23a regulates cardiomyocyte apoptosis by targeting manganese superoxide dismutase. Mol. Cells 40, 542-549 (2017).

109. Suzuki, A. et al. Active remodeling of the coronary arterial lesions in the late phase of Kawasaki disease: immunohistochemical study. Circulation 101 2935-2941 (2000)

110. Dahdah, N. Not just coronary arteritis, Kawasaki disease is a myocarditis, too. J. Am. Coll. Cardiol. 55, 1507 (2010).

111. Dionne, A. \& Dahdah, N. Myocarditis and Kawasaki disease. Int. J. Rheum. Dis. 21, 45-49 (2017).

112. Harada, M. et al. Histopathological characteristics of myocarditis in acute-phase Kawasaki disease. Histopathology 61, 1156-1167 (2012)

113. Yutani, C. et al. Cardiac biopsy of Kawasaki disease. Arch. Pathol. Lab. Med. 105, 470-473 (1981).

114. Takahashi, M. Myocarditis in Kawasaki syndrome. A minor villain? Circulation 79, 1398-1400 (1989).

115. Kao, C. H., Hsieh, K. S., Wang, Y. L., Wang, S. J. \& Yeh, S. H. The detection of ventricular dysfunction and carditis in children with Kawasaki disease using equilibrium multigated blood pooling ventriculography and 99Tcm-HMPAO-labelled WBC heart scans. Nucl. Med. Commun. 14, 539-543 (1993).

116. Printz, B. F. et al. Noncoronary cardiac abnormalities are associated with coronary artery dilation and with laboratory inflammatory markers in acute Kawasaki disease. J. Am. Coll. Cardiol. 57, 86-92 (2011).

117. Burns, J. C. Kawasaki disease update. Indian. J. Pediatr. 76, 71-76 (2009).

118. Miyake, T., Yokoyama, T., Shinohara, T., Seto, S. \& Oiki, M. Transient dilatation of the abdominal aorta in an infant with Kawasaki disease associated with thrombocytopenia. Acta Paediatr. Jpn. 37, 521-525 (1995). 
119. Canter, C. E.,Bower R. J \& Strauss, A. W. Atypical Kawasaki disease with aortic aneurysm. Pediatrics 68 885-888 (1981)

120. Amano, S. et al. General pathology of Kawasaki disease On the morphological alterations corresponding to the clinical manifestations. Acta Pathol. Jpn. 30, 681-694 (1980).

121. Takahashi, K., Oharaseki, T., Yokouchi, Y., Hiruta, N. \& Naoe, S. Kawasaki disease as a systemic vasculiti in childhood. Ann. Vasc. Dis. 3, 173-181 (2010).

122. Watanabe, T. Kidney and urinary tract involvement in Kawasaki disease. Int. J. Pediatr. 2013, 831834 (2013).

123. Watanabe, T. Clinical features of acute kidney injury in patients with Kawasaki disease. World J. Clin. Pediatr. 7, 83-88 (2018)

124. Ohshio, G. et al. High levels of IgA-containing circulating immune complex and secretory IgA in Kawasaki disease. Microbiol. Immunol. 31, 891-898 (1987).

125. Rowley, A. H. et al. Cloning the arterial IgA antibody response during acute Kawasaki disease. J. Immunol. 175, 8386-8391 (2005)

126. Rowley, A. H., Shulman, S. T., Spike, B. T., Mask, C. A. $\&$ Baker, S. C. Oligoclonal IgA response in the vascular wall in acute Kawasaki disease. J. Immunol. 166, 1334-1343 (2001)

127. Lee, Y. et al. IL-1 signaling is critically required in stromal cells in Kawasaki disease vasculitis mouse model: role of both IL- $1 \alpha$ and IL-1 $\beta$. Arterioscler. Thromb. Vasc. Biol. 35, 2605-2616 (2015).

128. Wakita, D. et al. Role of interleukin-1 signaling in a mouse model of Kawasaki disease-associated abdominal aortic aneurysm. Arterioscler. Thromb. Vasc. Biol. 36, 886-897 (2016).

129. Hoang, L. T. et al. Global gene expression profiling identifies new therapeutic targets in acute Kawasaki disease. Genome Med. 6, 541 (2014).

130. Fury, W. et al. Transcript abundance patterns in Kawasaki disease patients with intravenous immunoglobulin resistance. Hum. Immunol. 71 865-873 (2010).

131. Porritt, R. A et al. linterleukin-1-mediated sex differences in Kawasaki disease vasculitis development and response to treatment. Arterioscler. Thromb. Vasc. Biol. 40, 802-818 (2020).

132. Lee, Y. et al. Interleukin- $1 \beta$ is crucial for the induction of coronary artery inflammation in a mouse model of Kawasaki disease. Circulation 125, 1542-1550 (2012).

133. Ben-Sasson, S. Z. et al. IL-1 enhances expansion, effector function, tissue localization, and memory response of antigen-specific CD8 T cells. J. Exp. Med. 210, 491-502 (2013)

134. Hirao, J. \& Sugita, K. Circulating CD4+CD8+ T lymphocytes in patients with Kawasaki disease. Clin. Exp. Immunol. 111, 397-401 (1998)

135. Yilmaz, A. et al. Activated myeloid dendritic cells accumulate and co-localize with CD3 + T cells in coronary artery lesions in patients with Kawasaki disease. Exp. Mol. Pathol. 83, 93-103 (2007).

136. Bischoff, S. C. et al. Intestinal permeability-a new target for disease prevention and therapy. BMC Gastroenterol. 14, 189 (2014).

137. Miyake, T. et al. Small bowel pseudo-obstruction in Kawasaki disease. Pediatr. Radiol. 17, 383-386 (1987).

138. Yaniv, L., Jaffe, M. \& Shaoul, R. The surgica manifestations of the intestinal tract in Kawasaki disease. J. Pediatr. Surg. 40, e 1-e4 (2005).

139. Baker, A. L. et al. Associated symptoms in the ten days before diagnosis of Kawasaki disease. J. Pediatr. 154, 592-595 (2009)

140. Colomba, C. et al. Intestinal involvement in Kawasaki disease. J. Pediatr. 202, 186-193 (2018)

141. Fabi, M. et al. Gastrointestinal presentation of Kawasaki disease: a red flag for severe disease? PLoS One 13, e0202658 (2018).

142. Nagata, S., Yamashiro, Y., Maeda, M., Ohtsuka, Y. \& Yabuta, K. Immunohistochemical studies on small intestinal mucosa in Kawasaki disease. Pediatr. Res. 33, 557-563 (1993)

143. Noval Rivas, M. et al. Intestinal permeability and IgA provoke immune vasculitis linked to cardiovascular inflammation. Immunity 51, 508-521 (2019).

144. Tang, W. H. W. \& Hazen, S. L. The contributory role of gut microbiota in cardiovascular disease. J. Clin. Invest 124, 4204-4211 (2014).

145. Tang, W. H. W. \& Hazen, S. L. The gut microbiome and its role in cardiovascular diseases. Circulation 135, 1008-1010 (2017)

146. Takeshita, S., Kobayashi, I., Kawamura, Y., Tokutomi, T. $\&$ Sekine, I. Characteristic profile of intestinal microflora in Kawasaki disease. Acta Paediatr. 91, 783-788 (2002).

147. Yamashiro, Y., Nagata, S., Ohtsuka, Y., Oguchi, S $\&$ Shimizu, T. Microbiologic studies on the small intestine in Kawasaki disease. Pediatr. Res. 39 622-624 (1996)

148. Nagata, S. et al. Heat shock proteins and superantigenic properties of bacteria from the gastrointestinal tract of patients with Kawasaki disease. Immunology 128, 511-520 (2009).

149. Goldenberg, J. Z. et al. Probiotics for the prevention of pediatric antibiotic-associated diarrhea. Cochrane Database Syst. Rev. 12, CD004827 (2015).

150. Allen, S. J., Martinez, E. G., Gregorio, G. V. \& Dans, L. F. Probiotics for treating acute infectious diarrhoea. Cochrane Database Syst. Rev. 11, CD003048 (2010).

151. Karczewski, J. et al. Regulation of human epithelial tight junction proteins by Lactobacillus plantarum in vivo and protective effects on the epithelial barrier. Am. J. Physiol. Gastrointest. Liver Physiol. 298, G851-G859 (2010).

152. Han, X. et al. Lactobacillus rhamnosus GG prevents epithelial barrier dysfunction induced by interferongamma and fecal supernatants from irritable bowel syndrome patients in human intestinal enteroids and colonoids. Gut Microbes 10, 59-76 (2019).

153. Mack, D. R., Michail, S., Wei, S., McDougall, L. \& Hollingsworth, M. A. Probiotics inhibit enteropathogenic $\mathrm{E}$. coli adherence in vitro by inducing intestinal mucin gene expression. Am. J. Physiol. 276 G941-G950 (1999).

154. van Baarlen, P., Wells, J. M. \& Kleerebezem, M Regulation of intestinal homeostasis and immunity with probiotic lactobacilli. Trends Immunol. 34 208-215 (2013)

155. Belkacem, N. et al. Lactobacillus paracasei feeding improves immune control of influenza infection in mice. PLoS One 12, e0184976 (2017).

156. Belkacem, N., Bourdet-Sicard, R. \& Taha, M. K Lactobacillus paracasei feeding improves the control of secondary experimental meningococcal infection in flu-infected mice. BMC Infect. Dis. 18, 167 (2018).

157. Gebremariam, H. G. et al. Lactobacillus gasseri suppresses the production of proinflammatory cytokines in Helicobacter pylori-infected macrophages by inhibiting the expression of ADAM17. Front. Immunol. 10, $2326(2019)$

158. Downie, M. L. et al. Variability in response to intravenous immunoglobulin in the treatment of Kawasaki disease. J. Pediatr. 179, 124-130 (2016)

159. Dethlefsen, L. \& Relman, D. A. Incomplete recovery and individualized responses of the human distal gut microbiota to repeated antibiotic perturbation. Proc. Natl Acad. Sci. USA 108 (Suppl. 1), 4554-4561 (2011).

160. Modi, S. R., Collins, J. J. \& Relman, D. A. Antibiotics and the gut microbiota. J. Clin. Invest. 124 4212-4218 (2014)

161. Wheeler, M. L. et al. Immunological consequences of intestinal fungal dysbiosis. Cell Host Microbe 19 865-873 (2016)

162. Jernberg, C., Löfmark, S., Edlund, C. \& Jansson, J. K. Long-term ecological impacts of antibiotic administration on the human intestinal microbiota. ISME J. 1, 56-66 (2007).

163. Kinumaki, A. et al. Characterization of the gut microbiota of Kawasaki disease patients by metagenomic analysis. Front. Microbiol. 6, 824 (2015).

164. Audemard-Verger, A., Pillebout, E., Guillevin, L. Thervet, E. \& Terrier, B. IgA vasculitis (Henoch-Shönlein purpura) in adults: diagnostic and therapeutic aspects. Autoimmun. Rev. 14, 579-585 (2015).

165. Floege, J. \& Feehally, J. The mucosa-kidney axis in IgA nephropathy. Nat. Rev. Nephrol. 12, 147-156 (2016)

166. Coppo, R. The gut-kidney axis in IgA nephropathy: role of microbiota and diet on genetic predisposition. Pediatr. Nephrol. 33, 53-61 (2018).

167. Moja, P. et al. Is there IgA from gut mucosal origin in the serum of children with Henoch-Schönlein purpura? Clin. Immunol. Immunopathol. 86, 290-297 (1998).

168. Menikou, S., Langford, P. R. \& Levin, M. Kawasak disease: the role of immune complexes revisited. Front. Immunol. 10, 1156 (2019).

169. Oni, L. \& Sampath, S. Childhood IgA vasculitis (Henoch Schonlein Purpura)-advances and knowledge gaps. Front. Pediatr. 7, 257 (2019).

170. Oortwijn, B. D. et al. A pathogenic role for secretory IgA in IgA nephropathy. Kidney Int. 69, 1131-1138 (2006).

171. Nishimura, S. et al. A polymorphism in the promoter of the CD14 gene (CD14/-159) is associated with the development of coronary artery lesions in patients with Kawasaki disease. J. Pediatr. 143, 357-362 (2003).

172. Yoon, H. J. et al. Association of the CD14 gene $-159 \mathrm{C}$ polymorphism with progression of IgA nephropathy. J. Med. Genet. 40, 104-108 (2003).

173. Boyer, E. M., Turman, M. \& O'Neil, K. M. Partial response to anakinra in life-threatening HenochSchönlein purpura: case report. Pediatr. Rheumatol. Online J. 9, 21 (2011)

174. Chun, J. et al. NLRP3 localizes to the tubular epithelium in human kidney and correlates with outcome in IgA nephropathy. Sci. Rep. 6, 24667 (2016).

175. Meinzer, U. et al. Interleukin-1 targeting drugs in familial Mediterranean fever: a case series and a review of the literature. Semin. Arthritis Rheum. 41 265-271 (2011)

176. Lehman, T. J., Walker, S. M., Mahnovski, V. $\delta$ McCurdy, D. Coronary arteritis in mice following the systemic injection of group B Lactobacillus casei cell walls in aqueous suspension. Arthritis Rheum. 28 652-659 (1985).

177. Murata, H. Experimental candida-induced arteritis in mice. Relation to arteritis in the mucocutaneous lymph node syndrome. Microbiol. Immunol. 23, 825-831 (1979).

178. Nishio, H. et al. Nod1 ligands induce site-specific vascular inflammation. Arterioscler. Thromb. Vasc. Biol. 31, 1093-1099 (2011).

179. Hill, D. et al. The Lactobacillus casei group: history and health related applications. Front. Microbiol. 9 , 2107 (2018)

180. Lehman, T. J., Allen, J. B., Plotz, P. H. \& Wilder, R. L. Polyarthritis in rats following the systemic injection of Lactobacillus casei cell walls in aqueous suspension. Arthritis Rheum. 26, 1259-1265 (1983).

181. Noval Rivas, M. et al. CD8+ T cells contribute to the development of coronary arteritis in the Lactobacillus casei cell wall extract-induced murine model of Kawasaki disease. Arthritis Rheumatol. 69, 410-421 (2017).

182. Matundan, H. H. et al. Myocardial fibrosis after adrenergic stimulation as a long-term sequela in a mouse model of Kawasaki disease vasculitis. JCI Insight 4, e126279 (2019).

183. Abe, M. et al. IL-1-dependent electrophysiological changes and cardiac neural remodeling in a mouse model of Kawasaki disease vasculitis. Clin. Exp. Immunol. 199, 303-313 (2019).

184. Gorelik, M. et al. IL-1 receptor antagonist, anakinra, prevents myocardial dysfunction in a mouse model of Kawasaki disease vasculitis and myocarditis. Clin. Exp. Immunol. 198, 101-110 (2019).

185. Hui-Yuen, J. S., Duong, T. T. \& Yeung, R. S. M. TNF- $\alpha$ is necessary for induction of coronary artery inflammation and aneurysm formation in an animal model of Kawasaki disease. J. Immunol. 176. 6294-6301 (2006).

186. Lehman, T. J., Warren, R., Gietl, D., Mahnovski, V. \& Prescott, M. Variable expression of Lactobacillus casei cell wall-induced coronary arteritis: an animal model of Kawasaki's disease in selected inbred mouse strains. Clin. Immunol. Immunopathol. 48, 108-118 (1988).

187. Myones, B., Bathoria, J., Lehman, T. \& Shulman, S. in Kawasaki Disease: Proceedings of the 5th International Kawasaki Disease Symposium (ed. Kato, $\mathrm{H}$ et al.) 252-256 (Elsevier, 1995).

188. Maury, C. P., Salo, E. \& Pelkonen, P. Circulating interleukin- $1 \beta$ in patients with Kawasaki disease. N. Engl. J. Med. 319, 1670-1671 (1988).

189. Ohno, N. Chemistry and biology of angiitis inducer, Candida albicans water-soluble mannoprotein- $\beta$-glucan complex (CAWS). Microbiol. Immunol. 47, 479-490 (2003).

190. Takahashi, K. et al. Histopathological features of murine systemic vasculitis caused by Candida albicans extract-an animal model of Kawasaki disease. Inflamm. Res. 53, 72-77 (2004).

191. Oharaseki, T. et al. Recognition of alpha-mannan by dectin 2 is essential for onset of Kawasaki disease-like murine vasculitis induced by Candida albicans cell-wall polysaccharide. Mod. Rheumatol. 30, 350-357 (2020).

192. Yoshikane, Y. et al. JNK is critical for the development of Candida albicans-induced vascular lesions in a mouse model of Kawasaki disease. Cardiovasc. Pathol. 24, 33-40 (2015)

193. Nakamura, J. et al. Adeno-associated virus vector-mediated interleukin-10 induction prevents vascular inflammation in a murine model of Kawasak disease. Sci. Rep. 8, 7601 (2018). 
194. Sabat, R. et al. Biology of interleukin-10. Cytokine Growth Factor. Rev. 21, 331-344 (2010).

195. Ohno, N. Murine model of Kawasaki disease induced by mannoprotein-beta-glucan complex, CAWS, obtained from Candida albicans. Jpn. J. Infect. Dis. 57, S9-S10 (2004).

196. Miura, N. N. et al. IL-10 is a negative regulatory factor of CAWS-vasculitis in CBA/J mice as assessed by comparison with Bruton's tyrosine kinase-deficient CBA/N mice. J. Immunol. 183, 3417-3424 (2009).

197. Ohno, N. A murine model of vasculitis induced by fungal polysaccharide. Cardiovasc. Hematol. Agents Med. Chem. 6, 44-52 (2008).

198. Nagi-Miura, N., Adachi, Y. \& Ohno, N. Coronary arteritis induced by CAWS (Candida albicans water-soluble fraction) in various strains of mice. Nihon Ishinkin Gakkai Zasshi 49, 287-292 (2008).

199. Stock, A. T., Hansen, J. A., Sleeman, M. A. McKenzie, B. S. \& Wicks, I. P. GM-CSF primes cardiac inflammation in a mouse model of Kawasaki disease. J. Exp. Med. 213, 1983-1998 (2016).

200. Martinez, H. G. et al. Important role of CCR2 in a murine model of coronary vasculitis. BMC Immunol. 13, 56 (2012).

201. Miyabe, C. et al. Dectin-2-induced CCL2 production in tissue-resident macrophages ignites cardiac arteritis. J. Clin. Invest. 130, 3610-3624 (2019).

202. Stock, A. T., Jama, H. A., Hansen, J. A. \& Wicks, I. P. TNF and IL-1 play essential but temporally distinct roles in driving cardiac inflammation in a murine model of Kawasaki disease. J. Immunol. 202. 3151-3160 (2019)

203. Takahashi, K. et al. Administration of human immunoglobulin suppresses development of murine systemic vasculitis induced with Candida albicans water-soluble fraction: an animal model of Kawasak disease. Mod. Rheumatol. 20, 160-167 (2010).

204. Hashimoto, Y. et al. Interleukin-1 beta inhibition attenuates vasculitis in a mouse model of Kawasaki disease. J. Nippon. Med. Sch. 86, 108-116 (2019).

205. Anzai, F. et al. Crucial role of NLRP3 inflammasome in a murine model of Kawasaki disease. J. Mol. Cell Cardiol. 138, 185-196 (2019).

206. Ohashi, R. et al. Characterization of a murine model with arteritis induced by Nod 1 ligand, FK565: a comparative study with a CAWS-induced model. Mod. Rheumatol. 27, 1024-1030 (2017).

207. Motomura, Y. et al. Identification of pathogenic cardiac CD11 c+ macrophages in Nod1-mediated acute coronary arteritis. Arterioscler. Thromb. Vasc. Biol. 35, 1423-1433 (2015)

208. Iwata, M Shimozato, T Tokiwa, H \& Tsubura, E. Antipyretic activity of a human immunoglobulin preparation for intravenous use in an experimental model of fever in rabbits. Infect. Immun. 55, 547-554 (1987).

209. Ruiz de Souza, V et al Selective induction of interleukin-1 receptor antagonist and interleukin-8 in human monocytes by normal polyspecific IgG (intravenous immunoglobulin). Eur. J. Immunol. 25, 1267-1273 (1995).
210. Uehara, R. et al. Analysis of potential risk factors associated with nonresponse to initial intravenous immunoglobulin treatment among Kawasaki disease patients in Japan. Pediatr. Infect. Dis. J. 27, 155-160 (2008).

211. Tremoulet, A. H. et al. Infliximab for intensification of primary therapy for Kawasaki disease: a phase 3 randomised, double-blind, placebo-controlled trial. Lancet 383, 1731-1738 (2014)

212. Portman, M. A. et al. Etanercept with IVIg for acute Kawasaki disease: a randomized controlled trial. Pediatrics 143, e20183675 (2019).

213. Kobayashi, T. et al. Prediction of intravenous immunoglobulin unresponsiveness in patients with Kawasaki disease. Circulation 113, 2606-2612 (2006).

214. Sleeper, L. A. et al. Evaluation of Kawasaki disease risk-scoring systems for intravenous immunoglobulin resistance. J. Pediatr. 158, 831-835 (2011).

215. Kobayashi, T. et al. Efficacy of immunoglobulin plus prednisolone for prevention of coronary artery abnormalities in severe Kawasaki disease (RAISE study): a randomised, open-label, blinded-endpoints trial. Lancet 379, 1613-1620 (2012).

216. Lee, J., Kim, L. \& Choi, J. Revisiting the concept of targeting NFAT to control T cell immunity and autoimmune diseases. Front. Immunol. 9, 2747-2747 (2018).

217. Hamada, H. et al. Efficacy of primary treatment with immunoglobulin plus ciclosporin for prevention of coronary artery abnormalities in patients with Kawasaki disease predicted to be at increased risk of non-response to intravenous immunoglobulin (KAICA): a randomised controlled, open-label, blindedendpoints, phase 3 trial. Lancet 393, 1128-1137 (2019).

218. Tremoulet, A. H. et al. Rationale and study design for a phase I/lla trial of anakinra in children with Kawasak disease and early coronary artery abnormalities (the ANAKID trial). Contemp. Clin. Trials 48, 70-75 (2016).

219. Koné-Paut, I. et al. KAWAKINRA: a phase IIA multicenter trial to assess the efficacy, and safety of anakinra in patients with intravenous immunoglobulin-resistant Kawasaki disease [abstract OP0147]. Ann. Rheum. Dis. 78 (Suppl. 2), 149 (2019)

220. Cohen, S. et al. A child with severe relapsing Kawasaki disease rescued by IL-1 receptor blockade and extracorporeal membrane oxygenation. Ann. Rheum. Dis. 71, 2059-2061 (2012)

221. Shafferman, A., Birmingham, J. D. \& Cron, R. O. High dose anakinra for treatment of severe neonatal Kawasaki disease: a case report. Pediatr. Rheumatol. Online J. 12, 26 (2014)

222. Guillaume, M. P., Reumaux, H. \& Dubos, F. Usefulness and safety of anakinra in refractory Kawasaki disease complicated by coronary artery aneurysm. Cardiol. Young 28, 739-742 (2018).

223. Sánchez-Manubens, J. et al. A child with resistant Kawasaki disease successfully treated with anakinra: a case report. BMC Pediatr. 17, 102 (2017).
224. Blonz, G. et al. Severe late-onset Kawasaki disease successfully treated with anakinra. J. Clin. Rheumatol. 26, e42-e43 (2020).

225. Murata, K. et al. Calcineurin inhibitors exacerbate coronary arteritis via the MyD88 signalling pathway in a murine model of Kawasaki disease. Clin. Exp. Immunol. 190, 54-67 (2017).

226. Ohashi, R. et al. Etanercept suppresses arteritis in a murine model of Kawasaki disease: a comparative study involving different biological agents. Int. J. Vasc. Med. 2013, 543141 (2013).

227. Oharaseki, T. et al. The role of TNF-a in a murine model of Kawasaki disease arteritis induced with a Candida albicans cell wall polysaccharide. Mod. Rheumatol. 24 120-128 (2014).

228. Assas, B. M. et al. Anti-inflammatory effects of infliximab in mice are independent of tumour necrosis factor a neutralization. Clin. Exp. Immunol. 187 225-233 (2017).

229. Arora, T. et al. Differences in binding and effector functions between classes of TNF antagonists. Cytokine 45, 124-131 (2009).

230. Vos, A. C. et al. Anti-tumor necrosis factor- $\alpha$ antibodies induce regulatory macrophages in an Fc regiondependent manner. Gastroenterology 140, 221-230 (2011).

231. Mangan, M. S. J. et al. Targeting the NLRP3 inflammasome in inflammatory diseases. Nat. Rev. Drug. Discov. 17, 688 (2018).

232. Coll, R. C. et al. A small-molecule inhibitor of the NLRP3 inflammasome for the treatment of inflammatory diseases. Nat. Med. 21, 248-255 (2015)

233. Lehman, T. J., Allen, J. B., Plotz, P. H. \& Wilder, R. L. Lactobacillus casei cell wall-induced arthritis in rats: cell wall fragment distribution and persistence in chronic arthritis-susceptible LEW/N and -resistant F344/N rats. Arthritis Rheum. 27, 939-942 (1984).

\section{Acknowledgements}

The work of M.A. is supported by the NIH Grant R0 Al072726 and M.N.R. is supported by the NIH grant R01 HL139766.

\section{Author contributions}

Both authors contributed equally to all aspects of the manuscript.

\section{Competing interest}

The authors declare no competing interests.

\section{Peer review information}

Nature Reviews Rheumatology thanks Ho-Chang Kuo, Isabelle Kone-Paut and the other, anonymous, reviewer(s) for their contribution to the peer review of this work.

\section{Publisher's note}

Springer Nature remains neutral with regard to jurisdictional claims in published maps and institutional affiliations.

(c) Springer Nature Limited 2020 